\title{
Metabolic profiling of neocortical tissue discriminates Alzheimer's disease from mild cognitive impairment, high pathology controls, and normal controls
}

\section{Paniz Jasbi}

Arizona State University https://orcid.org/0000-0002-2129-3098

\section{Xiaojian Shi}

Yale University

\section{Ping Chu}

Midwestern University

Natalie Elliott

Midwestern University

Haley Hudson

Midwestern University

Douglas Jones

Midwestern University

\section{Geidy Serrano}

Banner Sun Health Research Institute

Thomas Beach

Banner Sun Health Research Institute

Li Liu

Arizona State University

\section{Garilyn Jentarra}

Midwestern University

Haiwei Gu ( $\nabla$ haiweigu@asu.edu )

Arizona State University https://orcid.org/0000-0002-7598-5022

\section{Research article}

Keywords: Alzheimer's disease, biomarkers, mass spectrometry, metabolomics, pathogenesis

Posted Date: November 20th, 2020

DOI: https://doi.org/10.21203/rs.3.rs-110947/v1 
License: (c) (i) This work is licensed under a Creative Commons Attribution 4.0 International License. Read Full License 


\section{Abstract \\ Background}

Alzheimer's disease (AD) is the most common cause of dementia, accounting for an estimated 60 to $80 \%$ of cases, and is the sixth-leading cause of death in the United States. While considerable advancements have been made in the clinical care of $A D$, it remains a complicated disorder that can be difficult to identify definitively in its earliest stages. Recently, mass spectrometry (MS)-based metabolomics has shown significant potential for elucidation of disease mechanisms and identification of therapeutic targets as well diagnostic and prognostic markers that may be useful in resolving some of the difficulties affecting clinical AD studies, such as effective stratification.

\section{Methods}

In this study, complementary gas chromatography- and liquid chromatography-MS platforms were used to detect and monitor 2,080 metabolites and features in 48 post-mortem tissue samples harvested from the superior frontal gyrus of male and female subjects. Samples were taken from four groups: 12 normal control (NC) patients, 12 cognitively normal subjects characterized as high pathology controls (HPC), 12 subjects with non-specific mild cognitive impairment (MCl), and 12 subjects with AD.

\section{Results}

Multivariate statistics informed the construction and cross-validation $(p<0.01)$ of partial least squaresdiscriminant analysis (PLS-DA) models defined by a 9-metabolite panel of disease markers (lauric acid, stearic acid, myristic acid, palmitic acid, palmitoleic acid, and four unidentified mass spectral features). Receiver operating characteristic analysis showed high predictive accuracy of the resulting PLS-DA models for discrimination of NC (97\%), HPC (92\%), MCl ( 96\%), and AD ( 96\%) groups. Pathway analysis revealed significant disturbances in lysine degradation, fatty acid metabolism, and the degradation of branched-chain amino acids. Network analysis showed significant enrichment of 11 enzymes, predominantly within the mitochondria.

\section{Conclusions}

The results expand basic knowledge of the metabolome related to $A D$ and reveal pathways that can be targeted therapeutically. This study also provides a promising basis for the development of larger multisite projects to validate these candidate markers in readily available biospecimens such as blood to enable the effective screening, rapid diagnosis, accurate surveillance, and therapeutic monitoring of $A D$.

\section{Background}


Alzheimer's disease (AD) is a neurodegenerative disorder marked primarily by cognitive decline and dementia [1, 2], in addition to the accumulation of extracellular amyloid $\beta(A \beta)$ plaques and intracellular neurofibrillary tau tangles $[3,4]$. AD is the most common cause of adult dementia, accounting for 60 to $80 \%$ of cases worldwide [5], and is sixth leading cause of death in the United States [6]. Currently, AD affects more than 5.8 million Americans [5, 7], with prevalence expected to triple by 2050 [8]. In the United States, total payments in 2020 for health care, long-term care, and hospice services are estimated to be $\$ 305$ billion [6]. Consequently, AD represents a significant threat to human health and exerts a substantial financial and societal impact.

Considerable advancements have been made in the ability to accurately diagnose $A D$, largely owing to the development of positron emission tomography (PET) scans for detection of plaques and tangles, as well as cerebral spinal fluid (CSF) and plasma tests for AD-associated biomarkers [9]. However, treatment of patients and appropriate evaluation of the outcomes of clinical studies remain complicated by the many unknowns involved in $A D$. Individuals with mild cognitive impairment $(\mathrm{MCl})$ may be in the early stages of $A D$ or may be affected by an unrelated disease process, confounding studies of early interventional treatments. In addition, some individuals with intermediate levels of AD-associated pathology (plaques and tangles) do not display cognitive deficits [10], indicating at least a partial disconnect between those specific pathologies and cognitive function. It is also common for AD to be found in conjunction with other pathologies such as synucleinopathy, TDP-43, or microinfarcts [11]. Therefore, the ability to more effectively stratify and subgroup individuals would likely produce clearer and more actionable results. Furthermore, provisional diagnosis relies on a combination of mental status testing, neuropsychological tests, interviews with friends and family, laboratory tests, and various brain imaging techniques such as magnetic resonance imaging (MRI), computerized tomography (CT), and PET [12-14]. These conventional diagnostic methods show low specificity against other dementias (70\%) and only moderate sensitivity (80\%) [15]. Additionally, these criteria are unable to capture early brain pathology that may predate symptoms by as much as 30 years [16]; without timely diagnosis, patients are less likely to access appropriate treatment options that may slow disease progression [17, 18]. Consequently, there is a critical need for highly sensitive and specific markers of $A D$ that may enable early disease detection as well as identification of potential drug targets, improved prognosis, and monitoring of therapeutic response.

A growing body of evidence suggests that perturbations in various metabolic pathways play a significant role in AD [11]. Most notably, the mitochondrial cascade hypothesis states that widespread mitochondrial metabolic dysfunction is a strong characteristic of $A D$ and plays a role in the accumulation of $A \beta$ plaques [12]. Furthermore, studies have also shown fatty acids to play an important role in AD pathology, exerting both protective and pathogenic effects [7, 13]. Alterations in glycerophospholipid [14] and phosphatidylcholine metabolism [15] have also been strongly linked to AD in previous studies utilizing metabolomics-based approaches. Metabolomics, the scientific study of metabolic composition and pathways present in biological systems, has facilitated the accurate characterization of various metabolomes for advances in disease classification, drug therapy, and biomarker discovery [16-18]. More specifically, mass spectrometry (MS) is an analytical approach in metabolomics that allows for the 
accurate detection and quantification of metabolites in biological samples [19-21]. Various forms of untargeted and targeted MS-based metabolomic assays have been implemented in an attempt to profile biochemical processes in AD pathology. Methods mainly include direct infusion-MS [22], ultrahigh resolution-MS enabled by liquid chromatography (LC)-Orbitrap [23], and gas chromatography (GC)-timeof-flight-MS [24], which were used in many previous studies for therapeutic drug targeting, disease characterization, and potential diagnostic biomarkers.

The current study employs hyphenated MS-based assays that combine both targeted and untargeted metabolomics approaches to detect aqueous metabolites, lipids, fatty acids, and bile acids, in addition to profiling unidentified features. A total of 2,080 metabolites/features were detected in 48 samples of superior frontal gyrus tissue taken from four groups of patients: normal control (NC), high pathology controls (HPC), mild cognitive impairment (MCl), and AD. Multivariate significance testing and model estimation constructed cross-validated partial least squares-discrimination analysis (PLS-DA) models confined to a highly predictive 9-metabolite panel of potential biomarkers capable of distinguishing each clinical group with high sensitivity and specificity. In conjunction with pathway and enrichment analyses, the current study corroborates findings of previous literature and adds to basic knowledge of the metabolome related to neurodegenerative decline as well as the behavioral symptoms of AD-induced dementia; this study offers a large-scale analysis of molecular alterations associated with $A D$ pathogenesis and progression, potentially supporting future drug development and prevention efforts. Importantly, this study provides clinically relevant candidate biomarkers capable of accurate post-mortem classification which may, eventually, prove useful to in vivo diagnosis and disease monitoring.

\section{Methods}

\section{Reagents}

Acetonitrile $(\mathrm{ACN})$, methanol $(\mathrm{MeOH})$, ammonium acetate $\left(\mathrm{NH}_{4} \mathrm{OAc}\right)$, acetic acid $(\mathrm{AcOH})$, and isopropanol (IPA), all LC-MS grade, were purchased from Fisher Scientific (Pittsburgh, PA). Ammonium hydroxide $\left(\mathrm{NH}_{4} \mathrm{OH}\right)$, methyl tert-butyl ether (MTBE), O-methylhydroxylamine hydrochloride (MeOX), and N-Methyl-N(tert-butyldimethylsilyl) trifluoroacetamide (MTBSTFA) were bought from Sigma-Aldrich (Saint Louis, MO). High performance LC grade chloroform $\left(\mathrm{CHCl}_{3}\right)$ was obtained from VWR (Radnor, PA). Deionized water was provided in-house by a water purification system from EMD Millipore (Billerica, MA). Phosphate buffered saline (PBS) was bought from GE Healthcare Life Sciences (Logan, UT). Standard compounds corresponding to measured aqueous metabolites/features were purchased from SigmaAldrich and Fisher Scientific. Lipid standards were purchased from Fisher Scientific, Sigma-Aldrich, and Avanti Polar Lipids (Alabaster, AL).

\section{Clinical samples}

Frozen tissue from the superior frontal gyrus of male and female subjects was obtained from the Arizona Study of Aging and Neurodegenerative Disorders/Brain and Body Donation Program at the Banner Sun 
Health Research Institute (BSHRI) in Sun City, Arizona [20]. Samples were collected under a previously approved institutional review board (IRB) protocol with broad consent for usage of biospecimens. All research protocols were conducted in accordance with the principles expressed in the Declaration of Helsinki. Subjects were divided into 4 groups by clinical status ( $n=12$ for all groups) based on assessment of post-mortem brain pathology and cognitive status before death. These groups were: normal control (NC) subjects with criteria not met for AD neuropathology, cognitively normal subjects with intermediate AD pathology characterized as high pathology controls (HPC), subjects with nonspecific mild cognitive impairment $(\mathrm{MCl})$ and intermediate $A D$ neuropathology, and subjects with dementia and high neuropathology with criteria met for $A D$. For definition of groups by AD pathology, the National Institute on Aging-Alzheimer's Association guidelines for the neuropathologic assessment of Alzheimer's disease were used [10]. Relevant clinical characteristics were provided by BSHRI for each subject such as age, sex, APOE genotype, post-mortem interval (PMI), Mini-Mental State Examination (MMSE), and Braak score. Additionally, measures of brain pathology including cerebral amyloid angiopathy (CAA), amyloid plaques and neurofibrillary tangles were taken from either a frontal area of the brain or a compilation of sampling from various brain regions.

\section{Targeted LC-MS/MS aqueous profiling}

For tissue lysates, $400 \mathrm{mg}$ pieces of frozen superior frontal gyrus were hand homogenized in $400 \mu \mathrm{L}$ of ice-cold sterile PBS containing a protease/phosphatase inhibitor cocktail (Halt, Thermo Scientific). Three samples for which less tissue was available were homogenized in equal ratios (weight to volume) of the PBS/inhibitor solution. Homogenized samples were sonicated on ice in a biosafety cabinet at a $40 \%$ amplitude for a total time of $1 \mathrm{~min}$, with alternating on/off sequences of $15 \mathrm{sec}$. Samples were then centrifuged for $30 \mathrm{~min}$ at $14,000 \mathrm{xg}$ at $4^{\circ} \mathrm{C}$. The supernatant and pellets were stored separately at $-80^{\circ} \mathrm{C}$ until analysis.

Prior to LC-MS/MS targeted measurement, frozen tissue supernatant samples were first thawed overnight under $4{ }^{\circ} \mathrm{C}$. Afterward, $50 \mu \mathrm{L}$ of each sample were placed in a $2 \mathrm{~mL}$ Eppendorf vial. The initial step for protein precipitation and metabolite extraction was performed by adding $500 \mu \mathrm{L} \mathrm{MeOH}$ and $50 \mu \mathrm{L}$ internal standard solution (containing $1,810.5 \mu \mathrm{M}^{13} \mathrm{C}_{3}$-lactate and $142 \mu \mathrm{M}^{13} \mathrm{C}_{5}$-glutamic acid). The mixture was then vortexed for $10 \mathrm{~s}$ and stored at $-20^{\circ} \mathrm{C}$ for $30 \mathrm{~min}$, followed by centrifugation at 14,000 RPM for $10 \mathrm{~min}$ at $4{ }^{\circ} \mathrm{C}$. The supernatants $(450 \mu \mathrm{L})$ were collected into new Eppendorf vials and dried using a CentriVap Concentrator. The dried samples were reconstituted in $150 \mu \mathrm{L}$ of $40 \% \mathrm{PBS} / 60 \% \mathrm{ACN}$ and centrifuged again at $14,000 \mathrm{RPM}$ at $4{ }^{\circ} \mathrm{C}$ for $10 \mathrm{~min}$. Afterward, $100 \mu \mathrm{L}$ of supernatant was collected from each sample into an LC autosampler vial for subsequent analysis. A pooled sample, which was a mixture of all experimental samples, was used as the quality control (QC) sample and injected once every 10 experimental samples.

The targeted LC-MS/MS method used here was modeled after that developed and used in a growing number of studies [25-30]. Briefly, all LC-MS/MS experiments were performed on an Agilent 1290 UPLC6490 QQQ-MS system. Each supernatant sample was injected twice, $10 \mu \mathrm{L}$ for analysis using negative 
ionization mode and $4 \mu \mathrm{L}$ for analysis using positive ionization mode. Both chromatographic separations were performed in hydrophilic interaction chromatography mode on a Waters XBridge BEH Amide column $(150 \times 2.1 \mathrm{~mm}, 2.5 \mu \mathrm{m}$ particle size, Waters Corporation, Milford, MA). The flow rate was $0.3 \mathrm{~mL} / \mathrm{min}$, auto-sampler temperature was kept at $4{ }^{\circ} \mathrm{C}$, and the column compartment was set to $40{ }^{\circ} \mathrm{C}$. The mobile phase was composed of Solvents $\mathrm{A}\left(10 \mathrm{mM} \mathrm{NH}_{4} \mathrm{OAc}, 10 \mathrm{mM} \mathrm{NH}_{4} \mathrm{OH}\right.$ in $\left.95 \% \mathrm{H}_{2} \mathrm{O} / 5 \% \mathrm{ACN}\right)$ and $\mathrm{B}(10 \mathrm{mM}$ $\mathrm{NH}_{4} \mathrm{OAc}, 10 \mathrm{mM} \mathrm{NH}_{4} \mathrm{OH}$ in $95 \% \mathrm{ACN} / 5 \% \mathrm{H}_{2} \mathrm{O}$ ). After an initial 1 min isocratic elution of $90 \% \mathrm{~B}$, the percentage of Solvent B decreased to $40 \%$ at $t=11 \mathrm{~min}$. The composition of Solvent B was maintained at $40 \%$ for $4 \mathrm{~min}$ ( $t=15 \mathrm{~min}$ ), after which the percentage of $B$ gradually went back to $90 \%$, to prepare for the next injection. The mass spectrometer was equipped with an electrospray ionization (ESI) source. Targeted data acquisition was performed in multiple-reaction-monitoring (MRM) mode. For targeted data acquisition, we monitored 118 and 160 MRM transitions in negative and positive mode, respectively (278 transitions in total). The whole LC-MS system was controlled by Agilent MassHunter Workstation software. The extracted MRM peaks were integrated using Agilent MassHunter Quantitative Data Analysis software.

\section{Targeted LC-MS/MS lipidomics}

Tissue samples were thawed under $4{ }^{\circ} \mathrm{C}$. Then, $200 \mu \mathrm{L} 10 \mathrm{x}$ diluted PBS and $80 \mu \mathrm{L}$ of $\mathrm{MeOH}$ containing $50 \mu \mathrm{M}$ PC $(17: 0,17: 0)$ and PG $(17: 0,17: 0)$ internal standards were added to $20 \mathrm{mg}$ of each thawed sample. A $1 / 2$ spoonful of stainless-steel micro beads was added to each sample, which was subsequently homogenized for $20 \mathrm{sec}$. Afterward, $400 \mu \mathrm{L}$ of MTBE was added to each sample (MTBE:MeOH: $\mathrm{H}_{2} \mathrm{O}=$ $10: 2: 5, \mathrm{v} / \mathrm{v} / \mathrm{v}$ ) and vortexed for $30 \mathrm{sec}$ followed by sonication in ice bath for $20 \mathrm{~min}$. Lastly, samples were centrifuged at 14,000 RPM to separate phases. The upper MTBE layer $(300 \mu \mathrm{L})$ was extracted, transferred to new $1.5 \mathrm{~mL}$ Eppendorf tubes, dried in a Vacufuge Plus Evaporator (Hamburg, Germany), and then reconstituted with $100 \mu \mathrm{L} 1: 1 \mathrm{CHCl}_{3} / \mathrm{MeOH}$. Each sample $(80 \mu \mathrm{L})$ was then transferred to a $\mathrm{LC}-\mathrm{MS}$ vial for LC-MS/MS targeted lipidomics analysis, while the remaining $20 \mu \mathrm{L}$ was pooled to create a QC sample.

For lipidomic profiling, all mass spectrometry experiments were done on an Agilent 1290 LC-6490 QQQMS (Santa Clara, CA), and $4 \mu \mathrm{L}$ was injected for positive ionization, whereas $6 \mu \mathrm{L}$ was used in negative ion mode injections. Both modes used reverse phase chromatography with a Waters XSelect HSS T3 column $(150 \times 2.1 \mathrm{~mm}, 2.5 \mu \mathrm{m}$ particle size; Waters Corporation, Milford, MA). The flow rate through the column was maintained at $0.3 \mathrm{~mL} / \mathrm{min}$. The mobile phase Solvent A was composed of $10 \mathrm{mM} \mathrm{NH}_{4} \mathrm{OAc}$ in $60 \% \mathrm{H} 2 \mathrm{O} / 40 \% \mathrm{ACN}$. Solvent B consisted of $10 \mathrm{mM} \mathrm{NH}_{4} \mathrm{OAC}$ in $90 \% \mathrm{IPA} / 10 \% \mathrm{ACN}$. An isocratic elution was used with $50 \%$ Solvent $B$ for 3 min before its percentage was gradually increased to $100 \%$ over the next 12 min. Following 10 min of continued $100 \%$ Solvent $B$, at $t=25$ min, the percent of $B$ was decreased gradually back to $50 \%$ to prepare for the next sample injection. The set of lipids covered in our LC-MS/MS lipidomics assay were the same as those in our previous study, and 357 lipids were selected from various lipid classes including fatty acids, glycerolipids, glycerophospholipids, sphingolipids, etc [31]. Lipid standards were used to test the MRM and retention time (RT) for each individual lipid.

\section{Untargeted GC-MS aqueous profiling}


The aqueous bottom layer $(180 \mu \mathrm{L})$ from the MTBE extraction described above was collected into a new Eppendorf tube for derivatization prior to untargeted metabolic profiling with GC-MS. The collected bottom layer was dried under vacuum at $37^{\circ} \mathrm{C}$ for $4 \mathrm{~h}$ using a CentriVap Concentrator (Labconco, Fort Scott, KS). The residues were first derivatized with $40 \mu \mathrm{L}$ of $20 \mathrm{mg} / \mathrm{mL}$ MeOX solution in pyridine under $60^{\circ} \mathrm{C}$ for $90 \mathrm{~min}$. Next, $60 \mu \mathrm{L}$ of MTBSTFA containing $d_{27}$-mysristic acid were added, and the mixture was incubated at $60^{\circ} \mathrm{C}$ for $30 \mathrm{~min}$. The samples were then vortexed for $30 \mathrm{sec}$, followed by centrifugation at 14,000 RPM for 10 min. Finally, $70 \mu \mathrm{L}$ of supernatant were collected from each sample into new glass vials for GC-MS analysis.

GC-MS conditions used here were mainly adopted from previous studies [19, 21]. Briefly, GC-MS experiments were performed on an Agilent 7820A GC-5977B MSD system (Santa Clara, CA) by injecting $1 \mu \mathrm{L}$ of prepared samples. Helium was used as the carrier gas with a constant flow rate of $1.2 \mathrm{~mL} / \mathrm{min}$. The separation of metabolites was achieved using an Agilent HP-5 ms capillary column $(30 \mathrm{~m} \times 250 \mu \mathrm{m} \times$ $0.25 \mu \mathrm{m})$. The column temperature was maintained at $60^{\circ} \mathrm{C}$ for $1 \mathrm{~min}$, increased at a rate of $10{ }^{\circ} \mathrm{C} / \mathrm{min}$ to $325^{\circ} \mathrm{C}$, and then held at this temperature for $10 \mathrm{~min}$. Mass spectral signals were recorded at an $\mathrm{m} / \mathrm{z}$ range of 50-600. Data extraction was performed using Agilent MassHunter Profinder software. A batch recursive feature extraction algorithm for small molecules was used, and peaks were filtered so that only peaks with absolute height $\geq 1,000$ counts were included. An RT tolerance of 0.10 min was established, and extraction was limited to the largest 1,000 compound groups. Results were filtered if the overall identification score was less than 75 .

\section{Long chain fatty acids (LCFAs)}

Weighed $20 \mathrm{mg}$ samples were added to separate Eppendorf tubes and prepared using the same protocol as that outlined for LC-MS/MS lipidomics. Derivatization was performed using the same protocol as that outlined for GC-MS untargeted profiling. For analysis of LCFAs, $60 \mu \mathrm{L}$ of supernatant was transferred to a glass vial for GC-MS analysis, while $20 \mu \mathrm{L}$ was pooled from each sample for QC analysis. GC-MS method was the same as that for GC-MS untargeted profiling.

\section{Short chain fatty acids (SCFAs)}

Frozen tissue samples were first thawed overnight under $4^{\circ} \mathrm{C}$. Afterward, $20 \mathrm{mg}$ of each sample was homogenized with $5 \mu \mathrm{L}$ hexanoic acid-3,3,3 (internal standard), $15 \mu \mathrm{L}$ sodium hydroxide ( $\mathrm{NaOH}[0.5 \mathrm{M}]$ ), and $500 \mu \mathrm{L}$ methanol (MeOH). Following storage at $-20^{\circ} \mathrm{C}$ for 20 min and centrifugation at $14,000 \mathrm{RPM}$ for $10 \mathrm{~min}, 450 \mu \mathrm{L}$ of supernatant were collected and sample pH was adjusted to 10 by adding $30 \mu \mathrm{L}$ of $\mathrm{NaOH}: \mathrm{H}_{2} \mathrm{O}(1: 4, \mathrm{v}: \mathrm{v})$. Samples were then dried, and they were measured using the same protocol as that outlined for GC-MS untargeted profiling.

\section{Bile acids}

Sample preparation techniques used here are well established and described in the previous literature [32-36]. Briefly, $50 \mathrm{mg}$ of each tissue sample were homogenized with methanol $(500 \mu \mathrm{L})$ and then vortexed for $10 \mathrm{sec}$. Samples were stored at $-20^{\circ} \mathrm{C}$ for $20 \mathrm{~min}$, followed by sonication in an ice bath for 
10 min and then centrifugation at 14,000 RPM for 15 min at $4{ }^{\circ} \mathrm{C}$. Supernatants $(450 \mu \mathrm{L})$ were vacuum dried and then reconstituted in $100 \mu \mathrm{L} \mathrm{MeOH} / \mathrm{H}_{2} \mathrm{O}(1: 1, \mathrm{v} / \mathrm{v})$. Each prepared sample $(2 \mu \mathrm{L})$ was injected into the LC-MS system (Agilent 1290 UPLC-6490 QQQ-MS) for analysis using negative ionization mode. The mobile phase was composed of $5 \mathrm{mM} \mathrm{NH}_{4} \mathrm{OAc}$ in $\mathrm{H}_{2} \mathrm{O}$ with $0.1 \% \mathrm{AcOH}(\mathrm{A})$ and $\mathrm{ACN}$ with $0.1 \% \mathrm{AcOH}$ (B). After a $1 \mathrm{~min}$ of isocratic elution of $75 \%$ Solvent $A$, the content percentage decreased to $5 \% \mathrm{~A}$ at $\mathrm{t}=$ $15 \mathrm{~min}$. The composition of Solvent A was then maintained at $5 \%$ for $10 \mathrm{~min}$, followed by an increase to $75 \%$ at $\mathrm{t}=25 \mathrm{~min}$. The MS parameters were the same as those reported for targeted LC-MS/MS aqueous profiling, except that 55 bile acids were included in the detection panel. Samples were spiked with mixtures of standard compounds in order to validate bile acid identities.

\section{Data analysis}

Following peak integration, data were normalized by tissue weight and lysate volume as appropriate. The data were $\log _{10}$-transformed and Pareto scaled prior to model construction. Univariate testing was performed using SPSS 22.0 (SPSS Inc., Chicago, IL). Multivariate statistical analyses were performed using open-source R software. Pathway and integrating enzyme enrichment analysis were performed and visualized using MetaboAnalyst v4.0 [37]. All data underlying the findings reported herein are provided as Separate Excel File $\mathbf{S 1 .}$

\section{Results}

\section{Clinical characteristics}

A total of 48 subjects were included in this study: NC ( $n=12), \operatorname{HPC}(n=12), \mathrm{MCl}(n=12)$, and $\operatorname{AD}(n=12)$. Table 1 shows the clinical information of subjects, while Table 2 shows the neuropathological characteristics of study subjects. Between AD patients and controls, subjects were age- and sex-matched such that no statistically significant difference was observed between groups $(p>0.05)$. Principal component analysis (PCA) conducted with all reliably detected metabolites between all groups was performed, and $95 \%$ confidence intervals were evaluated for potential outliers. The initial PCA revealed one outlier (sample 13, HPC subject), and this sample was therefore removed from subsequent analyses. 
Table 1

Clinical information of study subjects.

\begin{tabular}{|c|c|c|c|c|}
\hline & $\begin{array}{l}\text { Non-Demented } \\
\text { Normal Controls } \\
(n=12)\end{array}$ & $\begin{array}{l}\text { High } \\
\text { Pathology } \\
\text { Controls }(n= \\
\text { 12) }\end{array}$ & $\begin{array}{l}\text { Mild Cognitive } \\
\text { Impairment ( } n \\
=12)\end{array}$ & $\begin{array}{l}\text { Alzheimer's } \\
\text { Disease }(n \\
=12)\end{array}$ \\
\hline Age in years, mean (SD) & $79.7(12.2)$ & $90.3(5.1)$ & $88.1(8.6)$ & $81.3(8.3)$ \\
\hline Sex of subjects (male/female) & $7 / 5$ & $6 / 6$ & $8 / 4$ & $7 / 5$ \\
\hline $\begin{array}{l}\text { Post-mortem interval mean, } \\
\text { hours (SD) }\end{array}$ & $2.93(1.0)$ & $2.84(0.9)$ & $3.06(0.8)$ & $3.2(0.5)$ \\
\hline $\begin{array}{l}\text { Mini-mental state examination } \\
\text { score mean (SD) }\end{array}$ & $28.8(0.8)^{\star}$ & $27.6(1.4)$ & $26.0(3.4)^{\star \star}$ & $10.3(7.6)$ \\
\hline $\begin{array}{l}\text { APOE alleles: number of } \\
\text { subjects with each genotype } \\
2 / 3,2 / 4,3 / 3,3 / 4\end{array}$ & $0,0,8,4$ & $2,0,7,3$ & $0,1,9,2$ & $0,0,6,6$ \\
\hline \multicolumn{5}{|c|}{ *Scores not available for two subjects. } \\
\hline core not available for one & & & & \\
\hline
\end{tabular}

Table 2

Neuropathological characteristics of study subjects.

\begin{tabular}{|c|c|c|c|c|}
\hline & $\begin{array}{l}\text { Non- } \\
\text { Demented } \\
\text { Normal } \\
\text { Controls ( } n= \\
12)\end{array}$ & $\begin{array}{l}\text { High } \\
\text { Pathology } \\
\text { Controls ( } n \\
=12)\end{array}$ & $\begin{array}{l}\text { Mild } \\
\text { Cognitive } \\
\text { Impairment } \\
(n=12)\end{array}$ & $\begin{array}{l}\text { Alzheimer's } \\
\text { Disease ( } n \\
=12)\end{array}$ \\
\hline \multicolumn{5}{|l|}{$\begin{array}{l}\text { Frontal plaque and tangle scores } \\
\text { (number of subjects scoring none, } \\
\text { sparse, moderate, or frequent) }\end{array}$} \\
\hline Plaque score (based on CERAD) & $10,2,0,0$ & $0,0,0,12$ & $1,1,1,9$ & $0,0,0,12$ \\
\hline Tangle score & $11,0,0,0^{\dagger}$ & $9,3,0,0$ & $5,6,1,0$ & $0,2,1,9$ \\
\hline $\begin{array}{l}\text { Braak staging (number of subjects } \\
\text { stage } 0 \text { to stage } \mathrm{VI} \text { ) }\end{array}$ & $0,5,3,4,0,0$ & $0,0,1,2,9,0$ & $0,0,1,1,9,1,0$ & $0,0,0,0,6,6$ \\
\hline
\end{tabular}

\section{Case (MCl, AD) vs control (NC, HPC)}

To assess broad differences in metabolic profiles, groups were collapsed among case (MCI and $A D)$ and control (NC and HPC). Initial t-testing between case and control revealed two highly significant and predictive metabolites with $p<0.001$ and univariate area under curve (AUC) $>0.90$ : lauric acid and myristic acid. Box plots of these metabolites are given in Supporting Information Figure S1. In addition, a partial least squares-discriminant analysis (PLS-DA) model was constructed using levels of lauric and 
myristic acid, and receiver operating characteristic (ROC) analysis was conducted using model-implied values to assess performance. As shown in Fig. 1, the resulting PLS-DA scores plot showed appreciable separation between collapsed case and control groups, and ROC analysis by 100 -fold leave-one-out cross validation (LOOCV) showed an overall accuracy of $95 \%$, more than either metabolite individually.

\section{NC/HPC vs other groups}

To analyze differences among groups individually, we first compared metabolic profiles of NC vs $\mathrm{HPC} / \mathrm{MCl} / \mathrm{AD}$, and HPC vs NC/MCl/AD. Multivariate analysis of variance (MANOVA) testing and associated post-hoc comparisons were performed. As outlined in Table 3, four metabolites (lauric acid, myristic acid, stearic acid, palmitic acid) had false discovery rate (FDR) $q<0.05$ and were mostly predictive of NC and HPC groups. Normalized box plots of these metabolites between all groups are shown in Fig. 2. A PLS-DA model was constructed using levels of these four significant metabolites, and internal validation was performed using a 100 -iteration permutation test. More than $98 \%$ of total variance between the four study groups was explained by the first two components, and permutation testing revealed the model to be statistically sound. To assess the predictive performance of this unified biomarker panel, the resulting PLS-DA model was subjected to ROC analysis with 100 -fold LOOCV. ROC curves for each comparison are provided in Supporting Information Figure S2. Evaluation of model accuracy showed high classification performance for discrimination of NC samples (96.6\%, Figure S2A) and HPC samples (91.7\%, Figure S2B).

Table 3

Significant between-group metabolites as determined by MANOVA testing, focusing on NC and HPC groups.

\begin{tabular}{|lllll|}
\hline Metabolite & F-value & $p$-value & FDR $\boldsymbol{q}$ & Post-hoc tests \\
\hline Lauric acid & 23.360 & $3.98 \mathrm{E}-9$ & $8.29 \mathrm{E}-6$ & HPC - AD; NC - AD; HPC - MCl; NC - HPC; NC - MCl \\
\hline Myristic acid & 16.727 & $3.92 \mathrm{E}-7$ & $2.49 \mathrm{E}-4$ & HPC - AD; NC - AD; HPC - MCl; HPC - NC; NC - MCl \\
\hline Stearic acid & 9.911 & $4.34 \mathrm{E}-5$ & 0.036 & HPC - AD; NC - AD; HPC - MCl; HPC - NC; NC - MCl \\
\hline Palmitic acid & 9.133 & $8.56 E-5$ & 0.036 & HPC - AD; HPC - MCl; HPC - NC; NC - MCl \\
\hline
\end{tabular}

\section{$\mathrm{MCl}$ vs other groups}

In an effort to increase model performance for discrimination of the $\mathrm{MCl}$ subgroup (see Supporting Information Figure S2C), other groups were compared sequentially for identification of significant/predictive metabolites and model construction. Comparison of $\mathrm{MCl}$ and $\mathrm{NC}$ groups revealed lauric acid to be both highly significant $(p<0.001)$ and predictive (AUC $=0.993)$ (Supporting Information Figure S3). Comparison of $\mathrm{MCl}$ and HPC groups revealed four metabolites (myristic acid, palmitic acid, stearic acid, palmitoleic acid) to have AUC $>0.90$ and FDR $q<0.05$ (Fig. 3), while comparison of $\mathrm{MCl}$ and AD groups revealed four unidentified features (from untargeted GC-MS) with AUC $>0.80$ and $q<0.05$ (Fig. 3). Candidate metabolites for the classification of $\mathrm{MCl}$ and $\mathrm{HPC}$ samples were used to construct an 
independent PLS-DA model, while candidate markers for $\mathrm{MCl}$ discrimination from AD samples were ported to construct a separate PLS-DA model. ROC analysis showed a predictive accuracy of $96.6 \%$ for the identification of $\mathrm{MCl}$ samples from high pathology controls (Fig. 4A). Meanwhile, ROC analysis of the PLS-DA model constructed using levels of four unidentified features with an average AUC $\sim 0.811$ showed an appreciable improvement in accuracy relative to each univariate AUC; classification accuracy of the PLS-DA model exhibited an AUC = 0.917 for discrimination of $\mathrm{MCl}$ and $\mathrm{AD}$ groups (Fig. 4B).

\section{AD vs other groups}

Relevant groups were also compared to AD samples for enhanced identification of disease. Univariate ROC analysis and independent samples t-testing of NC and AD samples showed lauric acid to be highly significant $(p<0.001)$ and predictive (AUC > 0.99) (see Supporting Information Figure S4). For classification of $A D$ samples from high pathology controls, t-testing revealed the unified biomarker panel in Fig. 2 as being significantly altered between groups $(p<0.01)$ and to have high predictive potential (AUC > 0.90). Direct comparisons between AD and HPC groups for these metabolites are visualized as box plots in Supporting Information Figure S5. For discrimination of these groups, an additional PLS-DA model was constructed using the aforementioned candidate markers, and ROC analysis revealed high predictive accuracy (94.8\%) for discrimination of AD and HPC samples (Fig. 5).

\section{Correlation analysis of candidate markers and clinical/neuropathological characteristics}

To assess relevant associations between the set of candidate markers and measures of brain pathology and disease progression, a correlation analysis was performed, and measures of association strength and significance were evaluated. A visualization of association strength between correlation variables is given in Fig. 6. Full details regarding magnitude of association $(r)$ and significance of association $(p)$ can be found in Supporting Information Table S1. In total, 4 associations had $r>0.5$ or $<-0.5$ and $p<0.05$. Lauric acid showed strong, significant associations with frontal plaque $(r=-0.598, p<0.001)$, total plaque $(r=-0.579, p<0.001)$, total tangle $(r=-0.507, p<0.001)$, and Braak score $(r=-0.539, p<0.001)$.

Furthermore, correlation analysis between age, sex, APOE allele, and all 2,080 reliably detected metabolites/features was also performed; no association was observed to be both strongly correlated ( $r=$ $-0.5-0.5)$ and statistically significant $(p>0.05)$.

\section{Pathway and enrichment analyses of metabolic data}

Subjects were grouped as case ( $\mathrm{MCl}$ and $\mathrm{AD}$ ) and control (NC and HPC) for analysis of significantly impacted pathways in response to Alzheimer's progression. Pathway analysis was conducted using KEGG database searches and metabolite intensities (Fig. 7). Three pathways were observed to have large impact coefficients (>0.5): (1) linoleic acid metabolism, (2) alanine, aspartate and glutamate metabolism, and (3) arginine and proline metabolism. Importantly, three pathways were found to be significantly 
affected $(p<0.05)$ as a result of increased AD pathogenesis. Namely, those were lysine degradation, fatty acid metabolism, and valine, leucine, and isoleucine degradation.

Subjects were dichotomously grouped as case/control, and enrichment analysis was conducted using a library containing 912 metabolic sets that are predicted to be changed in the case of dysfunctional enzymes using a genome-scale network model of human metabolism (Fig. 8). Eleven enzymes were found to be significantly enriched $(p<0.05)$. Notably, seven of those were mitochondrial enzymes indicated against the background of mitochondrial pathways. Full results of the enzyme enrichment analysis are displayed in Supporting Information Table S2.

\section{Discussion}

For the last two decades, significant innovations in MS-based metabolic profiling and analysis of disease-related alterations have been made and, in doing so, these efforts have borne highly sensitive and valuable diagnostic information [38-40]. In the current study, we explored a combination of targeted and untargeted metabolic profiling in addition to advanced multivariate statistical analysis for the discovery of sensitive and specific metabolite biomarkers for rapid AD classification post-mortem. To capture the diversity of metabolites involved in $A D$ pathobiology, we have used this particular method to detect 2,080 metabolites of the superior frontal gyrus from many biologically relevant metabolic pathways. Our multi-step biomarker selection, model construction, and cross validation have demonstrated the robust diagnostic power of this metabolic profiling method in this study of $48 \mathrm{NC}, \mathrm{HPC}$, $\mathrm{MCl}$ and $\mathrm{AD}$ subjects. Additionally, we have applied complementary LC/GC-MS approaches for enhanced monitoring of the metabolome related to $A D$ and, cumulatively, our results show clinically relevant disturbances in energy metabolism and substrate utilization.

The metabolite profiling approach presented in this study determined 5 fatty acids capable of discriminating AD patients from NC and HPC samples with an average AUC of $97 \%$. Recent metabolomics studies have also shown perturbations in fatty acid metabolism across differing Alzheimer pathologies. It was found that the dysregulation of sphingolipids and glycerophospholipids, long-chain fatty acids, and unsaturated fatty acids have been associated with $\operatorname{AD}[7,24,41]$. Similarly, significant disturbances in fatty acid metabolism were also observed in the current study $(p=0.008)$. Medium-chain fatty acids like lauric acid, which is found in high levels in coconut oil, have been proposed as possible nutritional therapies for the treatment of cognitive decline [42-44], and a significant difference in lauric acid was also observed in the $A D$ and $N C$ groups in this study. The markedly reduced levels of these fatty acids observed in our AD subjects could be linked to the impaired glucose metabolism that is welldocumented in AD patients [45-47]. Declines in the levels of the identified fatty acids in conjunction with decreased glucose metabolism might suggest that $\beta$-oxidation of fatty acids, which is generally low in the brain, is being upregulated to support the energy needs of the brain in AD patients. Supplementation of the fatty acids that can be rapidly metabolized might help support the energy needs of the brain, potentially ameliorating symptoms. This might account for the data cited in the above referenced reports which suggest that the addition of lauric acid to the diet (via coconut oil), may improve some symptoms 
in AD patients. Lauric acid is known to cross the blood-brain barrier [48], and dietary lauric acid might therefore be accessible as an energy source for the brain [49]. The results of the current study warrant further investigation of the therapeutic potential of lauric acid for the treatment and prevention of $A D$.

Previous studies have shown evidence for brain glucose dysregulation in $A D$ as characterized by higher brain tissue glucose concentration, reduced glycolytic flux, and lower GLUT3 expression as a function of increasing $A D$ pathogenesis $[45,50,51]$. Interestingly, the literature has shown involvement of the Warburg effect in non-tumor disease processes [52] and, in the context of AD, loss of brain aerobic glycolysis as a function of normal human aging is associated with increased tau deposition in preclinical $A D[53,54]$. In addition, previous results have shown impaired hypothalamic insulin signaling to be associated with elevated BCAA levels in a mouse model of AD [55], while defects in BCAA metabolism have in-turn been shown to drive primary $A D$ neuropathology [55]. A prospective cohort study of over 22,000 participants found significant associations between circulating BCAAs and risk of incident dementia and $A D$ [56]. It has been shown that defects in BCAA metabolism, and subsequent accumulation, can lead to the phosphorylation of tau proteins and the incidence of AD [57]. Other studies have found post-translational modifications to the stabilizing tau proteins, which were induced by lysine residues. It has been proposed that these modifications may play an integral role in the pathobiology of tau protein [58]. Our pathway analysis also revealed similar results with a significant degradation of lysine $(p=0.007)$ and BCAAs $(p=0.025)$, potentially signifying the underlying pathophysiology of AD. Given the recent failure of numerous billion-dollar clinical trials targeting traditionally hypothesized $A D$ mechanisms such as reduced acetylcholine, $A \beta$ plaques/neurofibrillary tangles, and tau protein [59], our enzyme and pathway enrichment results further corroborate previous evidence of widespread mitochondrial dysfunction concomitant with $A \beta$ pathology and $A D$ progression [59-61], providing compelling evidence for mitochondrial bioenergetics as a novel therapeutic target for preventing/slowing the onset/progression of $A D$.

Overall, our findings led to an integrated hypothesis describing the pathophysiology of AD in Fig. 9 and are conceptualized with respect to the widespread mitochondrial dysfunction observed in our results. As can be seen, with increased $A D$ pathogenicity, significant metabolic reprogramming is observed. Specifically, a decrease in aerobic glycolysis is followed by a shift toward degradation of BCAA for energy production, mostly associated with HPC and $\mathrm{MCl}$ subgroups. With even greater disease progression, further metabolic reprogramming is observed; fatty acids are progressively utilized for generation of ATP via increased $\beta$-oxidation activity and generation of $\mathrm{FADH}_{2}$ and $\mathrm{NADH}$ for oxidative phosphorylation in the electron transport chain. Preference for fatty acid substrates was most pronounced in the $\mathrm{MCl}$ and $\mathrm{AD}$ subgroups.

Additionally, we evaluated levels of 4 unidentified features with $p<0.05$ and $F C>2$, which informed the construction of independent PLS-DA models for enhanced classification of $A D$ from $\mathrm{MCl}$ samples. The combination of these 4 features had a diagnostic sensitivity and specificity of $84.1 \%$ and $86.3 \%$, respectively $(A U C=0.917)$. Although accurate tests for AD pathology with high severity are currently available (i.e., PET amyloid and tau, CSF amyloid and tau, plasma tau), diagnostic tests useful for 
intermediate (MCl) and low (HPC) pathology levels are still lacking. In this study, diagnosis of HPC and $\mathrm{MCl}$ subgroups was achieved with more than $90 \%$ overall AUC. Realization of these findings in plasma or CSF may inform clinical trial selection and study stratification, enable mass screening, and indicate viable therapeutic targets.

\section{Strengths and limitations}

A major strength of the study lies in the well-characterized BSHRI cohort [20] with measures of cognitive status and neuropathological examination at death. Furthermore, inclusion of traditionally understudied $\mathrm{HPC}$ and $\mathrm{MCl}$ groups allowed for the metabolic characterization of asymptomatic individuals with ADconsistent pathology and non-AD individuals with cognitive decline, respectively. Cumulatively, our panel of candidate markers shows potential for classification of individuals with early brain pathology and other dementias, facilitating enhanced post-mortem diagnosis. Furthermore, if validated in readily available biospecimens with minimally invasive sample collection, this novel panel of candidate markers may enable early disease diagnosis and enhanced treatment options. Additionally, we applied six distinct metabolomics assays encompassing complementary GC and LC techniques to ensure maximal coverage of the brain metabolome and were able to monitor more than 2,000 metabolites and features. Given the known benefit of complementary MS platforms for elucidation of $A D$ pathology $[18,62,63]$, our largescale multi-platform metabolomics approach utilizing both targeted and untargeted profiling enables comprehensive pathway and enzyme analysis, a key strength of this study to previous literature.

The main limitation of this study is the relatively small sample size. Moreover, our samples were taken cross-sectionally and therefore cannot infer longitudinal changes in metabolite information over time. Also, samples were only taken from a single brain region; inferences to other AD-associated brain structures are unknown. Nevertheless, conventional power was achieved for all biomarker analyses $(\beta<$ $0.2)$, and models were internally validated $(p<0.01)$. Our results warrant further investigation in a larger sample with serial cognitive assessments taken during life as well as tissue samples collected from distinct brain regions both resistant and vulnerable to AD pathology in order to monitor possible differential changes between tissue types.

\section{Conclusions}

This study is part of a growing body of literature in which MS-based metabolomics methods have been utilized for disease biomarker discovery and accurate classification [28-30,37]. We performed comparisons of brain tissue metabolites from $\mathrm{AD}$ patients, $\mathrm{MCl}$ samples, as well as high pathology and normal controls using both targeted LC-MS/MS metabolic profiling and an untargeted GC-MS approach [64]. Our results demonstrate significant alterations in a variety of the metabolites, mainly fatty acids,

which are characteristic of different groups. Furthermore, we evaluated the performance of 4 unidentified metabolic features and, through multivariate model construction, achieved an overall classification performance of $>90 \%$ for comparison of $A D$ and $\mathrm{MCl}$ patients, which has the potential to fulfill critical clinical needs [65]. Application of bioinformatic methods expanded basic knowledge of the metabolome 
related to $A D$ and showed decreased glycolytic function with increased degradation of BCAAs and $\beta$ oxidation of fatty acids associated with increased AD pathogenicity. Results of our fold change analysis, significance testing, and pathway analysis indicate metabolites and pathways previously shown to be crucial in immune response inhibition [66] and increased AD severity [67]. Likewise, the metabolites and associated metabolic pathways and enzymes identified in this study may inform the development of new therapeutic treatments for AD. In addition, this study provides a strong basis for larger multi-site projects to validate our findings across different population groups and further advances the development of improved clinical care for $A D$ patients.

\section{Abbreviations}

Acetic acid $(\mathrm{AcOH})$; acetonitrile $(\mathrm{ACN})$; ammonium acetate $\left(\mathrm{NH}_{4} \mathrm{OAc}\right)$; ammonium hydroxide $\left(\mathrm{NH}_{4} \mathrm{OH}\right)$; Alzheimer's disease (AD); amyloid $\beta(A B)$; area under curve (AUC); Banner Sun Health Research Institute (BSHRI); branched-chain amino acid (BCAA); cerebral amyloid angiopathy (CAA); cerebrospinal fluid (CSF); chloroform $\left(\mathrm{CHCl}_{3}\right)$; computerized tomography (CT); electrospray ionization (ESI); gas chromatography (GC); high pathology control (HPC); isopropanol (IPA); institutional review board (IRB); leave-one-out cross validation (LOOCV); liquid chromatography (LC); long-chain fatty acid (LCFA); magnetic resonance imaging (MRI); mass spectrometry (MS); methanol (MeOH); methyl tert-butyl ether (MTBE); mild cognitive impairment (MCl); Mini-Mental State Examination (MMSE); multiple reaction monitoring (MRM); multivariate analysis of variance (MANOVA); N-Methyl-N-(tert-butyldimethylsilyl) trifluoroacetamide (MTBSTFA), normal control (NC); O-methylhydroxylamine hydrochloride (MeOX); partial least squares-discriminant analysis (PLS-DA); phosphate buffered saline (PBS); positron emission tomography (PET); post-mortem interval (PMI); principal component analysis (PCA); quality control (QC); receiver operating characteristic $(\mathrm{ROC})$; retention time (RT)

\section{Declarations}

\section{Ethics approval and consent to participate}

All research protocols were conducted in accordance with the principles expressed in the Declaration of Helsinki.

\section{Consent for publication}

Samples were collected under a previously approved IRB protocol with broad consent for usage of biospecimens.

\section{Availability of data and materials}

All data analyzed during this study are included in its supplementary information files.

\section{Competing interests}


The authors declare that they have no competing interests.

\section{Funding}

Support from the College of Health Solutions (Jumpstart) at Arizona State University to HG is gratefully acknowledged. This work was also supported by funding to GJ from the Arizona Alzheimer's Consortium (funded by the Arizona Department of Health Services, Grant No. CTR040636) and matching funds from Midwestern University. Human tissues used in this study were provided at no cost by the Banner Sun Health Research Institute (BSHRI) Brain and Body Donation Program (BBDP). Support for recovery of tissues by the BBDP and provision of subject data collected by the Arizona Study of Aging and Neurodegenerative Disorders (AZSAND) is provided by the following grants: National Institute of Neurological Disorders and Stroke, U24 NS072026 National Brain and Tissue Resource for Parkinson's Disease and Related Disorders; National Institute on Aging, P30 AG19610 Arizona Alzheimer's Disease Core Center; Arizona Department of Health Services, Arizona Alzheimer's Consortium; Arizona Biomedical Research Commission, Arizona Parkinson's Disease Consortium; Michael J. Fox Foundation for Parkinson's Research. The funders had no role in study design, data collection and analysis, decision to publish, or preparation of the manuscript.

\section{Authors' contributions}

GJ and HG designed the research. GS and TB facilitated sample acquisition. PC, NE, and HH processed tissue samples. PJ prepared samples for biomarkers assays. XS analyzed the samples. PJ integrated the mass spectral data; PJ, LL, and HG analyzed the data. DJ, TB, and GJ assisted in the interpretation of results. PJ, TB, GJ, and HG wrote the manuscript. All authors read and approved the final manuscript.

\section{Acknowledgements}

Not applicable.

\section{Author's information}

Not applicable.

\section{References}

1. Qiu C, De Ronchi D, Fratiglioni L. The epidemiology of the dementias: An update. Curr Opin Psychiatry. 2007;20: 380-385. doi:10.1097/YC0.0b013e32816ebc7b

2. Costa AC, Joaquim HPG, Forlenza O V., Gattaz WF, Talib LL. Three plasma metabolites in elderly patients differentiate mild cognitive impairment and Alzheimer's disease: a pilot study. Eur Arch Psychiatry Clin Neurosci. 2019. doi:10.1007/s00406-019-01034-9

3. Hölscher C. Development of beta-amyloid-induced neurodegeneration in Alzheimer's disease and novel neuroprotective strategies. Rev Neurosci. 2005;16: 181-212. 
doi:10.1515/REVNEURO.2005.16.3.181

4. Belbin O, Brown $\mathrm{K}$, Shi H, Medway $\mathrm{C}$, Abraham R, Passmore P, et al. A multi-center study of ACE and the risk of late-onset Alzheimer's disease. J Alzheimer's Dis. 2011;24: 587-597. doi:10.3233/JAD2011-101914

5. Yi L, Liu W, Wang Z, Ren D, Peng W. Characterizing Alzheimer's disease through metabolomics and investigating anti-Alzheimer's disease effects of natural products. Ann N Y Acad Sci. 2017;1398: 130-141. doi:10.1111/nyas. 13385

6. Alzheimer's Association Report. 2020 Alzheimer's disease facts and figures. Alzheimer's Dement. 2020;16: 391-460. doi:10.1002/alz.12068

7. Snowden SG, Ebshiana AA, Hye A, An Y, Pletnikova O, O'Brien R, et al. Association between fatty acid metabolism in the brain and Alzheimer disease neuropathology and cognitive performance: $\mathrm{A}$ nontargeted metabolomic study. PLoS Med. 2017;14: 1-19. doi:10.1371/journal.pmed.1002266

8. Hendrix JA, Bateman RJ, Brashear HR, Duggan C, Carrillo MC, Bain LJ, et al. Challenges, solutions, and recommendations for Alzheimer's disease combination therapy. Alzheimer's Dement. 2016;12: 623-630. doi:10.1016/j.jalz.2016.02.007

9. Hane FT, Robinson M, Lee BY, Bai O, Leonenko Z, Albert MS. Recent Progress in Alzheimer's Disease Research, Part 3: Diagnosis and Treatment. J Alzheimer's Dis. 2017;57: 645-665. doi:10.3233/JAD160907

10. Hyman BT, Phelps CH, Beach TG, Bigio EH, Cairns NJ, Carrillo MC, et al. National Institute on AgingAlzheimer's Association guidelines for the neuropathologic assessment of Alzheimer's disease. Alzheimer's Dement. 2012;8: 1-13. doi:10.1016/j.jalz.2011.10.007

11. Nelson PT, Abner EL, Schmitt FA, Kryscio RJ, Jicha GA, Smith CD, et al. Modeling the association between 43 different clinical and pathological variables and the severity of cognitive impairment in a large autopsy cohort of elderly persons. Brain Pathol. 2010;20: 66-79. doi:10.1111/j.17503639.2008.00244.x

12. Kang S, Jeong H, Baek JH, Lee SJ, Han SH, Cho HJ, et al. PiB-PET Imaging-Based Serum Proteome Profiles Predict Mild Cognitive Impairment and Alzheimer's Disease. J Alzheimer's Dis. 2016;53: 1563-1576. doi:10.3233/JAD-160025

13. Seyfried NT, Dammer EB, Swarup V, Nandakumar D, Duong DM, Yin L, et al. A Multi-network Approach Identifies Protein-Specific Co-expression in Asymptomatic and Symptomatic Alzheimer's Disease. Cell Syst. 2017;4: 60-72.e4. doi:10.1016/j.cels.2016.11.006

14. McKhann G, Drachman D, Folstein M, Katzman R, Price D, Stadlan EM. Clinical diagnosis of Alzheimer's disease: Report of the NINCDS-ADRDA work group $\star$ under the auspices of department of health and human services task force on Alzheimer's disease. Neurology. 1984;34: 939-944. doi:10.1212/wnl.34.7.939

15. Iverson DJ, Gronseth GS, Reger MA, Classen S, Dubinsky RM, Rizzo M. Practice parameter update: Evaluation and management of driving risk in dementia: Report of the quality standards 
subcommittee of the American academy of neurology. Neurology. 2010;74: 1316-1324. doi:10.1212/WNL.0b013e3181da3b0f

16. Sancesario GM, Bernardini S. Alzheimer's disease in the omics era. Clin Biochem. 2018;59: 9-16. doi:10.1016/j.clinbiochem.2018.06.011

17. Roberts R, Knopman DS. Classification and epidemiology of MCl. Clin Geriatr Med. 2013;29: 753772. doi:10.1016/j.cger.2013.07.003

18. González-Domínguez R, Sayago A, Fernández-Recamales Á. Metabolomics in Alzheimer's disease: The need of complementary analytical platforms for the identification of biomarkers to unravel the underlying pathology. J Chromatogr B Anal Technol Biomed Life Sci. 2017;1071: 75-92. doi:10.1016/j.jchromb.2017.02.008

19. Gu H, Gowda GAN, Neto FC, Opp MR, Raftery D. RAMSY: Ratio Analysis of Mass Spectrometry to Improve Compound Identification. Anal Chem. 2013;85: 10771-10779. doi:10.1021/ac4019268

20. Beach TG, Adler CH, Sue LI, Serrano G, Shill HA, Walker DG, et al. Arizona Study of Aging and Neurodegenerative Disorders and Brain and Body Donation Program. Neuropathology. 2015;35: 354-389. doi:10.1111/neup.12189

21. Kind T, Wohlgemuth G, Lee DY, Lu Y, Palazoglu M, Shahbaz S, et al. FiehnLib: Mass Spectral and Retention Index Libraries for Metabolomics Based on Quadrupole and Time-of-Flight Gas Chromatography/Mass Spectrometry. Anal Chem. 2009;81: 10038-10048. doi:10.1021/ac9019522

22. González-Domínguez R, García-Barrera T, Gómez-Ariza JL. Using direct infusion mass spectrometry for serum metabolomics in Alzheimer's disease. Anal Bioanal Chem. 2014;406: 7137-7148. doi:10.1007/s00216-014-8102-3

23. Lin S, Liu H, Kanawati B, Liu L, Dong J, Li M, et al. Hippocampal metabolomics using ultrahighresolution mass spectrometry reveals neuroinflammation from Alzheimer's disease in CRND8 mice. Anal Bioanal Chem. 2013;405: 5105-5117. doi:10.1007/s00216-013-6825-1

24. Wang G, Zhou Y, Huang FJ, Tang HD, Xu XH, Liu JJ, et al. Plasma metabolite profiles of Alzheimer's disease and mild cognitive impairment. J Proteome Res. 2014;13: 2649-2658. doi:10.1021/pr5000895

25. Zhu J, Djukovic D, Deng L, Gu H, Himmati F, Chiorean EG, et al. Colorectal Cancer Detection Using Targeted Serum Metabolic Profiling. J Proteome Res. 2014;13: 4120-4130. doi:10.1021/pr500494u

26. Carroll PA, Diolaiti D, McFerrin L, Gu H, Djukovic D, Du J, et al. Deregulated Myc Requires MondoA/Mlx for Metabolic Reprogramming and Tumorigenesis. Cancer Cell. 2015;27: 271-285. doi:10.1016/J.CCELL.2014.11.024

27. Gu H, Zhang P, Zhu J, Raftery D. Globally Optimized Targeted Mass Spectrometry: Reliable Metabolomics Analysis with Broad Coverage. Anal Chem. 2015;87: 12355-12362. doi:10.1021/acs.analchem.5b03812

28. Gu H, Carroll PA, Du J, Zhu J, Neto FC, Eisenman RN, et al. Quantitative Method to Investigate the Balance between Metabolism and Proteome Biomass: Starting from Glycine. Angew Chemie Int Ed. 2016;55: 15646-15650. doi:10.1002/anie.201609236 
29. Li R, Grimm SA, Mav D, Gu H, Djukovic D, Shah R, et al. Transcriptome and DNA Methylome Analysis in a Mouse Model of Diet-Induced Obesity Predicts Increased Risk of Colorectal Cancer. Cell Rep. 2018;22: 624-637. doi:10.1016/j.celrep.2017.12.071

30. Buas MF, Gu H, Djukovic D, Zhu J, Onstad L, Reid BJ, et al. Candidate serum metabolite biomarkers for differentiating gastroesophageal reflux disease, Barrett's esophagus, and high-grade dysplasia/esophageal adenocarcinoma. Metabolomics. 2017;13: 23. doi:10.1007/s11306-016-1154y

31. Eghlimi R, Shi X, Hrovat J, Xi B, Gu H. Triple Negative Breast Cancer Detection Using LC-MS/MS Lipidomic Profiling. J Proteome Res. 2020;19: 2367-2378. doi:10.1021/acs.jproteome.0c00038

32. Li CY, Dempsey JL, Wang D, Lee SW, Weigel KM, Fei Q, et al. PBDEs altered gut microbiome and bile acid homeostasis in male C57BL/6 mice. Drug Metab Dispos. 2018;46: 1226-1240. doi:10.1124/dmd.118.081547

33. Dempsey JL, Wang D, Siginir G, Fei Q, Raftery D, Gu H, et al. Pharmacological Activation of PXR and CAR Downregulates Distinct Bile Acid-Metabolizing Intestinal Bacteria and Alters Bile Acid Homeostasis. Toxicol Sci. 2019;168: 40-60. doi:10.1093/toxsci/kfy271

34. Ginos BNR, Navarro SL, Schwarz Y, Gu H, Wang D, Randolph TW, et al. Circulating bile acids in healthy adults respond differently to a dietary pattern characterized by whole grains, legumes and fruits and vegetables compared to a diet high in refined grains and added sugars: A randomized, controlled, crossover feeding stud. Metabolism. 2018;83: 197-204.

doi:10.1016/J.METABOL.2018.02.006

35. Gutierrez D, Weinstock A, Antharam VC, Gu H, Jasbi P, Shi X, et al. Antibiotic-induced gut metabolome and microbiome alterations increase the susceptibility to Candida albicans colonization in the gastrointestinal tract. FEMS Microbiol Ecol. 2019;96: fiz187. doi:10.1093/femsec/fiz187

36. Scoville DK, Li CY, Wang D, Dempsey JL, Raftery D, Mani S, et al. Polybrominated Diphenyl Ethers and Gut Microbiome Modulate Metabolic Syndrome-Related Aqueous Metabolites in Mice. Drug Metab Dispos. 2019;47: 928-940. doi:10.1124/dmd.119.086538

37. Chong J, Soufan O, Li C, Caraus I, Li S, Bourque G, et al. MetaboAnalyst 4.0: Towards more transparent and integrative metabolomics analysis. Nucleic Acids Res. 2018;46: W486-W494. doi:10.1093/nar/gky310

38. Gowda GN, Raftery D. Biomarker discovery and translation in metabolomics. Curr Metabolomics. 2013;1: 227-240. Available: https://www.ingentaconnect.com/content/ben/cmb/2013/00000001/00000003/art00004

39. Andrisic L, Dudzik D, Barbas C, Milkovic L, Grune T, Zarkovic N. Short overview on metabolomics approach to study pathophysiology of oxidative stress in cancer. Redox Biol. 2018;14: 47-58. doi:10.1016/j.redox.2017.08.009

40. Lawal O, Ahmed WM, Nijsen TME, Goodacre R, Fowler SJ. Exhaled breath analysis: a review of 'breath-taking' methods for off-line analysis. Metabolomics. 2017;13: 1-16. doi:10.1007/s11306017-1241-8 
41. Varma VR, Oommen AM, Varma S, Casanova R, An Y, Andrews RM, et al. Brain and blood metabolite signatures of pathology and progression in Alzheimer disease: A targeted metabolomics study. PLoS Med. 2018;15: 1-31. doi:10.1371/journal.pmed.1002482

42. Nafar F, Clarke JP, Mearow KM. Coconut oil protects cortical neurons from amyloid beta toxicity by enhancing signaling of cell survival pathways. Neurochem Int. 2017;105: 64-79. doi:10.1016/j.neuint.2017.01.008

43. Chatterjee P, Fernando M, Fernando B, Dias CB, Shah T, Silva R, et al. Potential of coconut oil and medium chain triglycerides in the prevention and treatment of Alzheimer's disease. Mech Ageing Dev. 2020;186: 111209. doi:10.1016/j.mad.2020.111209

44. de la Rubia Ortí JE, García-Pardo MP, Drehmer E, Sancho Cantus D, Julián Rochina M, Aguilar MA, et al. Improvement of Main Cognitive Functions in Patients with Alzheimer's Disease after Treatment with Coconut Oil Enriched Mediterranean Diet: A Pilot Study. J Alzheimer's Dis. 2018;65: 577-587. doi:10.3233/JAD-180184

45. Chen Z, Zhong C. Decoding Alzheimer's disease from perturbed cerebral glucose metabolism: Implications for diagnostic and therapeutic strategies. Prog Neurobiol. 2013;108: 21-43. doi:10.1016/j.pneurobio.2013.06.004

46. Szablewski L. Glucose Transporters in Brain: In Health and in Alzheimer's Disease. J Alzheimer's Dis. 2016;55: 1307-1320. doi:10.3233/JAD-160841

47. Burns CM, Chen K, Kaszniak AW, Lee W, Alexander GE, Bandy D, et al. Higher serum glucose levels are associated with cerebral hypometabolism in Alzheimer regions. Neurology. 2013;80: 1557-1564. doi:10.1212/WNL.0b013e31828f17de

48. Spector R. Fatty Acid Transport Through the Blood-Brain Barrier. J Neurochem. 1988;50: 639-643. doi:10.1111/j.1471-4159.1988.tb02958.x

49. Fernando WMADB, Martins IJ, Goozee KG, Brennan CS, Jayasena V, Martins RN. The role of dietary coconut for the prevention and treatment of Alzheimer's disease: Potential mechanisms of action. $\mathrm{Br}$ J Nutr. 2015;114: 1-14. doi:10.1017/S0007114515001452

50. Butterfield DA, Halliwell B. Oxidative stress, dysfunctional glucose metabolism and Alzheimer disease. Nat Rev Neurosci. 2019;20: 148-160. doi:10.1038/s41583-019-0132-6

51. An Y, Varma VR, Varma S, Casanova R, Dammer E, Pletnikova O, et al. Evidence for brain glucose dysregulation in Alzheimer's disease. Alzheimer's Dement. 2018;14: 318-329. doi:10.1016/j.jalz.2017.09.011

52. Chen Z, Liu M, Li L, Chen L. Involvement of the Warburg effect in non-tumor diseases processes. J Cell Physiol. 2017;233: 2839-2849. doi:10.1002/jcp.25998

53. Vlassenko AG, Gordon BA, Goyal MS, Su Y, Blazey TM, Durbin TJ, et al. Aerobic glycolysis and tau deposition in preclinical Alzheimer's disease. Neurobiol Aging. 2018;67: 95-98. doi:10.1016/j.neurobiolaging.2018.03.014

54. Goyal MS, Vlassenko AG, Blazey TM, Su Y, Couture LE, Durbin TJ, et al. Loss of Brain Aerobic Glycolysis in Normal Human Aging. Cell Metab. 2017;26: 353-360.e3. 
doi:10.1016/j.cmet.2017.07.010

55. Ruiz HH, Chi T, Shin AC, Lindtner C, Hsieh W, Ehrlich M, et al. Increased susceptibility to metabolic dysregulation in a mouse model of Alzheimer's disease is associated with impaired hypothalamic insulin signaling and elevated BCAA levels. Alzheimer's Dement. 2016;12: 851-861. doi:10.1016/j.jalz.2016.01.008

56. Tynkkynen J, Chouraki V, van der Lee SJ, Hernesniemi J, Yang Q, Li S, et al. Association of branchedchain amino acids and other circulating metabolites with risk of incident dementia and Alzheimer's disease: A prospective study in eight cohorts. Alzheimer's Dement. 2018;14: 723-733. doi:10.1016/j.jalz.2018.01.003

57. Li H, Ye D, Xie W, Hua F, Yang Y, Wu J, et al. Defect of branched-chain amino acid metabolism promotes the development of Alzheimer's disease by targeting the mTOR signaling. Biosci Rep. 2018;38: BSR20180127. doi:10.1042/BSR20180127

58. Kontaxi C, Piccardo P, Gill AC. Lysine-directed post-translational modifications of tau protein in Alzheimer's disease and related tauopathies. Front Mol Biosci. 2017;4. doi:10.3389/fmolb.2017.00056

59. Albensi BC. Dysfunction of mitochondria: Implications for Alzheimer's disease. Int Rev Neurobiol. 2019;145: 13-27. doi:10.1016/bs.irn.2019.03.001

60. Wilkins HM, Swerdlow RH. Relationships Between Mitochondria and Neuroinflammation: Implications for Alzheimer's Disease. Curr Top Med Chem. 2015;16: 849-857. doi:10.2174/1568026615666150827095102

61. Swerdlow RH. Mitochondria and Mitochondrial Cascades in Alzheimer's Disease. J Alzheimer's Dis. 2018;62: 1403-1416. doi:10.3233/JAD-170585

62. Fiehn 0 . Metabolomics by gas chromatography-mass spectrometry: Combined targeted and untargeted profiling. Curr Protoc Mol Biol. 2016;114: 30.4.32. doi:10.1002/0471142727.mb3004s114

63. González-Domínguez R, González-Domínguez Á, Sayago A, Fernández-Recamales Á. Mass spectrometry-based metabolomic multiplatform for Alzheimer's disease research. Methods in Molecular Biology. Humana Press Inc.; 2018. pp. 125-137. doi:10.1007/978-1-4939-7704-8_8

64. Klavins K, Koal T, Dallmann G, Marksteiner J, Kemmler G, Humpel C. The ratio of phosphatidylcholines to lysophosphatidylcholines in plasma differentiates healthy controls from patients with Alzheimer's disease and mild cognitive impairment. Alzheimer's Dement Diagnosis, Assess Dis Monit. 2015;1: 295-302. doi:10.1016/j.dadm.2015.05.003

65. Sun C, Gao M, Wang F, Yun Y, Sun Q, Guo R, et al. Serum metabolomic profiling in patients with Alzheimer Disease and Amnestic Mild Cognitive Impairment by GC/MS. Biomed Chromatogr. 2020 [cited 24 May 2020]. doi:10.1002/bmc.4875

66. Godyń J, Jończyk J, Panek D, Malawska B. Therapeutic strategies for Alzheimer's disease in clinical trials. Pharmacol Reports. 2016;68: 127-138. doi:10.1016/j.pharep.2015.07.006

67. Blennow K, de Leon MJ, Zetterberg H. Alzheimer's disease. Lancet. 2006;368: 387-403. doi:10.1016/S0140-6736(06)69113-7 
Figures

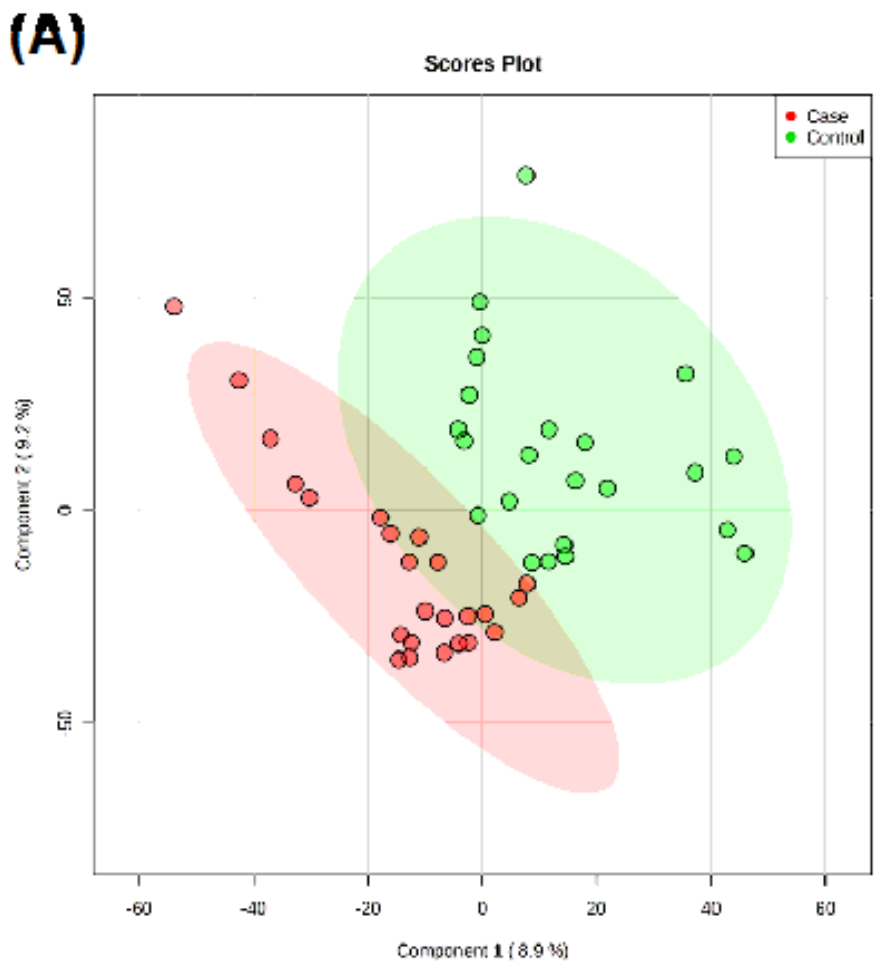

(B)

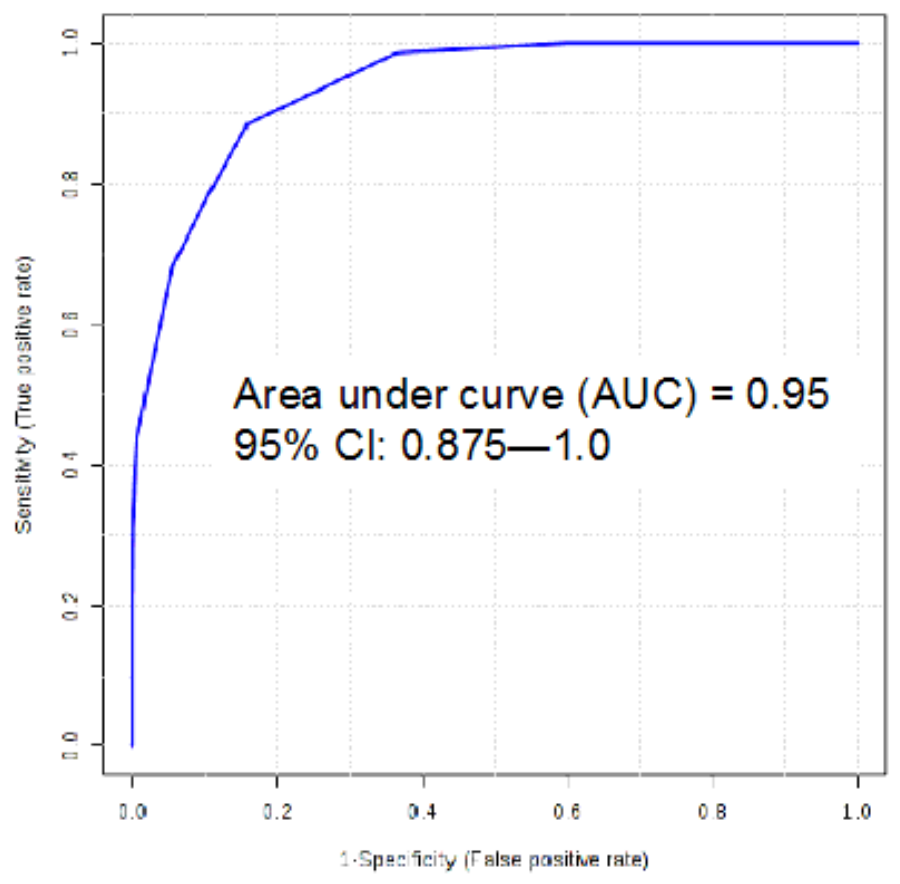

Figure 1

PLS-DA and ROC analysis of case (MCl and $A D)$ and control (NC and HPC) constructed using levels of lauric acid and myristic acid: (A) Scores plot of PLS-DA model $(R 2 X=0.593, R 2 Y=0.814, R 2 Q=0.701)$ and (B) ROC analysis of model-implied values showing $A U C=0.95$. 

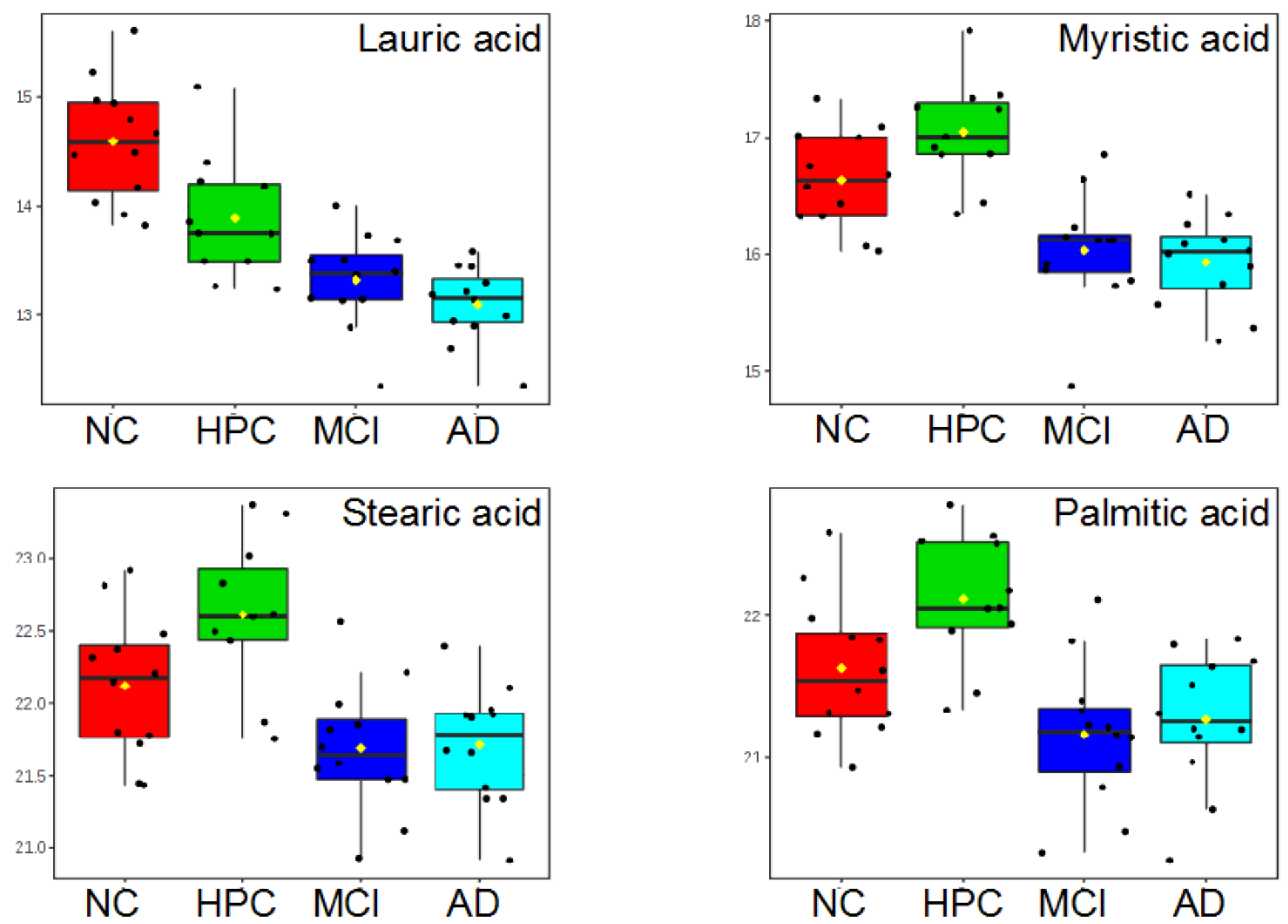

Figure 2

Box plots of four metabolites found to be significant (Bonferroni $p<0.05$ ) by MANOVA testing. Data were log10-transformed and Pareto scaled prior to plotting. 
Myristic acid

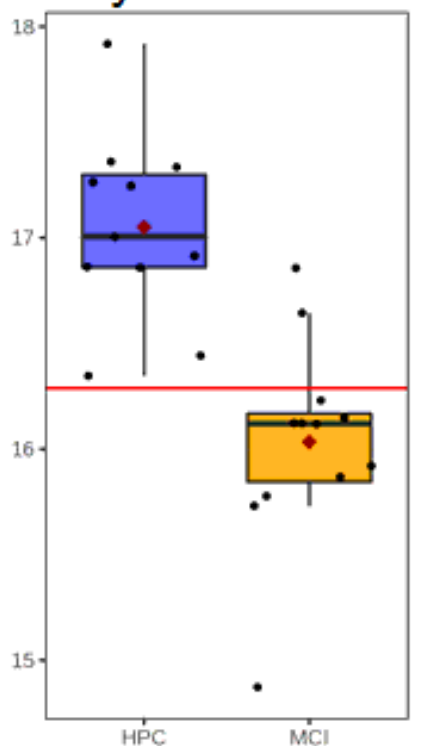

AUC $=0.970$ $p=3.96 \mathrm{E}-5$
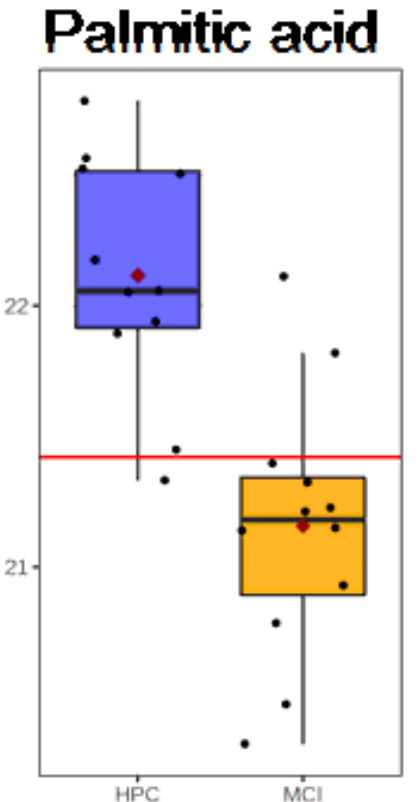

$\mathrm{AUC}=0.932$

$p=1.09 E-4$
Stearic acid

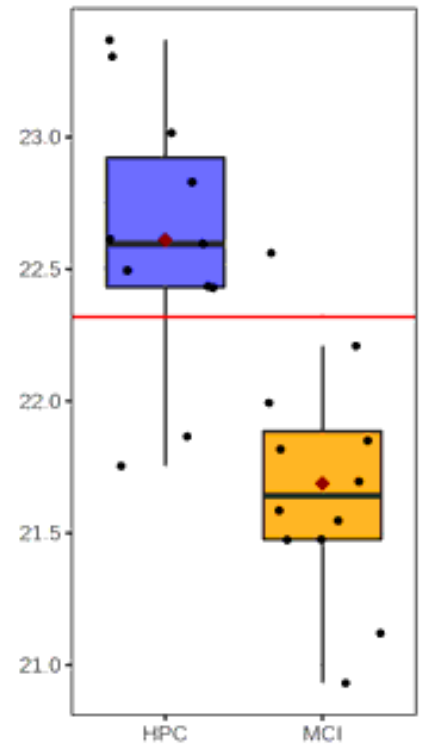

$\mathrm{AUC}=0.917$

$\mathrm{p}=1.52 \mathrm{E}-4$
Palmitoleic acid

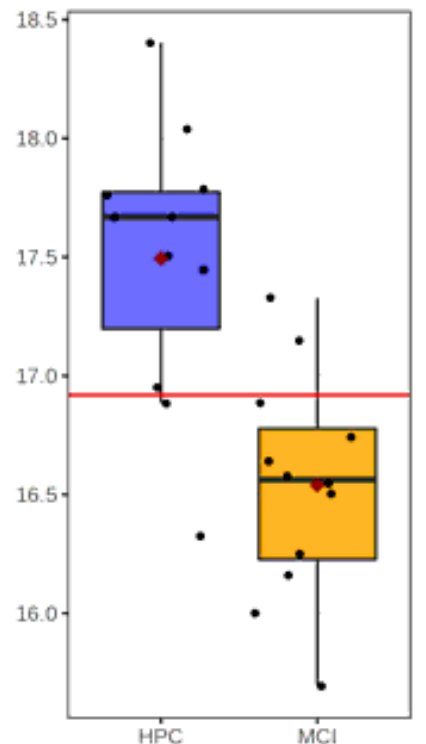

AUC $=0.902$

$p=2.75 E-4$

\section{1@14.3080147.1@20.774873.1@27.8681272.2@20.9320}

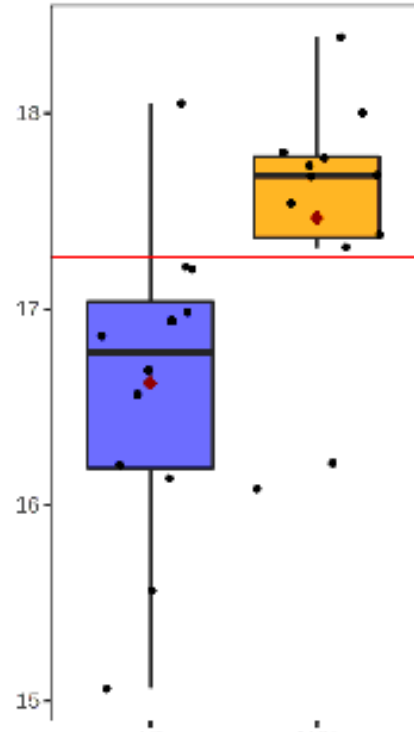

Á $\quad$ W'CI

$\mathrm{AUC}=0.813$

$p=0.011$

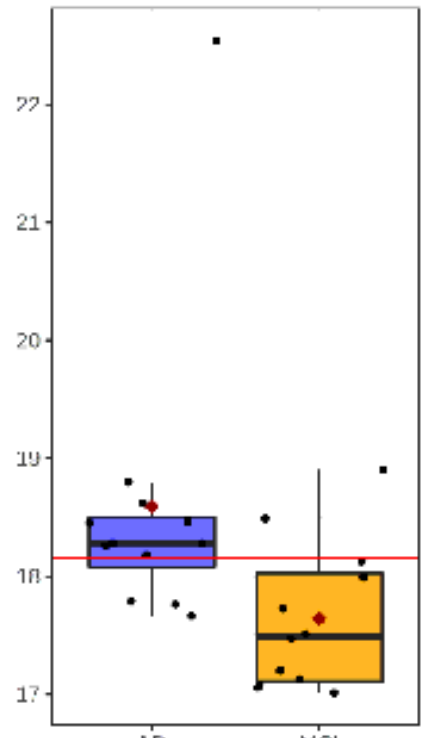

$\mathrm{AUC}=0.813$

$p=0.032$

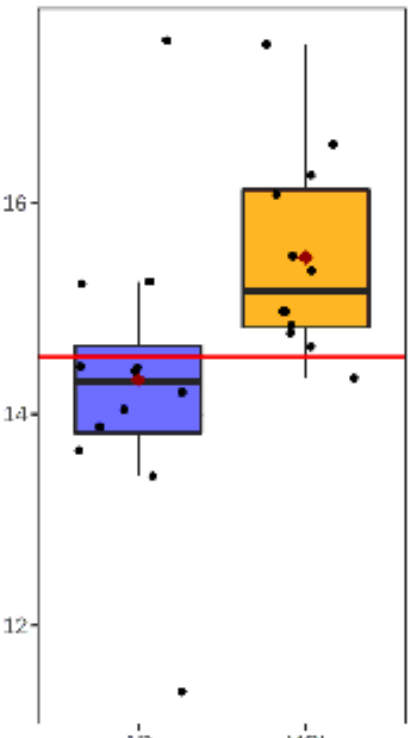

$\mathrm{AUC}=0.813$

$p=0.028$

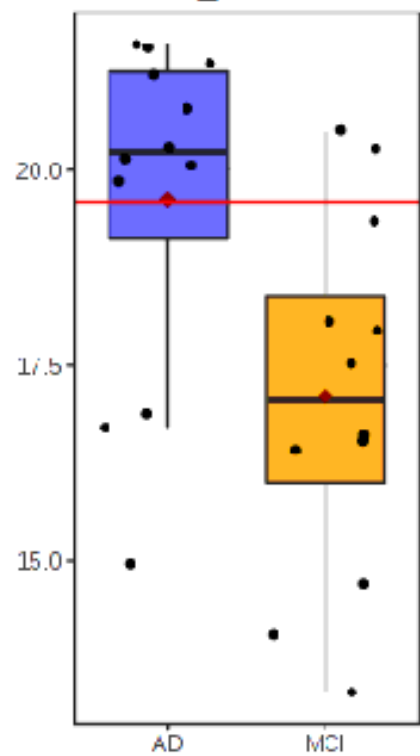

$\mathrm{AUC}=0.806$

$p=0.012$

Figure 3

Top row: Box plots of myristic acid, palmitic acid, stearic acid, and palmitoleic acid with high predictive accuracy (AUC > 0.90) and significance (FDR q < 0.05) in univariate ROC analysis and t-testing between $\mathrm{HPC}$ and $\mathrm{MCl}$ groups. Bottom row: Box plots of four unidentified features with good predictive accuracy (AUC > 0.80) and significance (FDR q < 0.05) in univariate ROC analysis and t-testing between $\mathrm{MCl}$ and AD groups. 
(A)

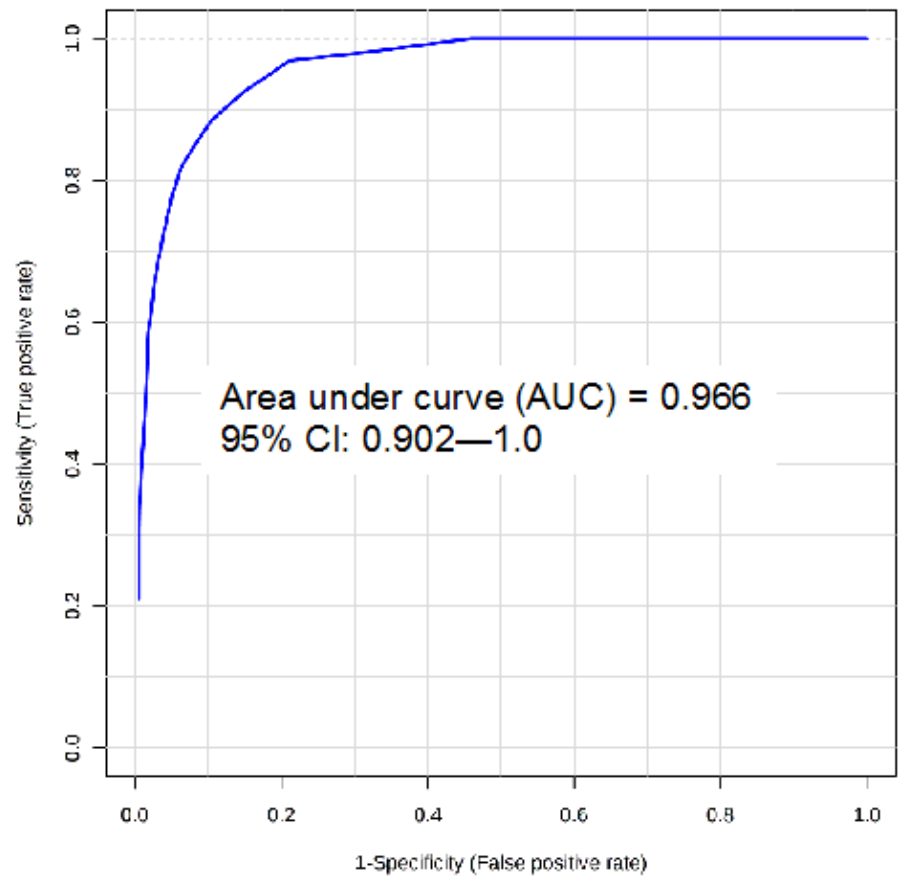

(B)

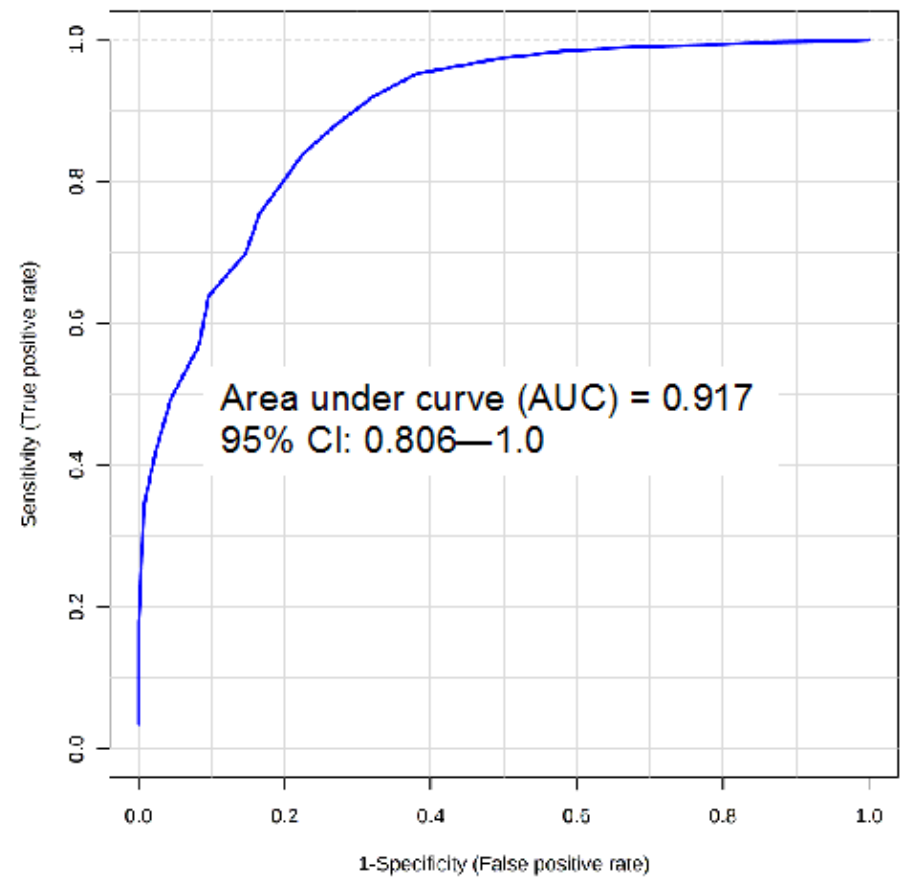

Figure 4

ROC analysis of classification performance by 100-fold LOOCV: (A) The PLS-DA model constructed using levels of myristic acid, palmitic acid, stearic acid, and palmitoleic acid for classification of $\mathrm{MCl}$ and $\mathrm{HPC}$ samples (AUC $=0.966)$, and $(B)$ The PLS-DA model constructed using levels of four significant $(p<0.05)$ unidentified features for classification of $\mathrm{MCl}$ and $\mathrm{AD}$ samples (AUC $=0.917$ ).
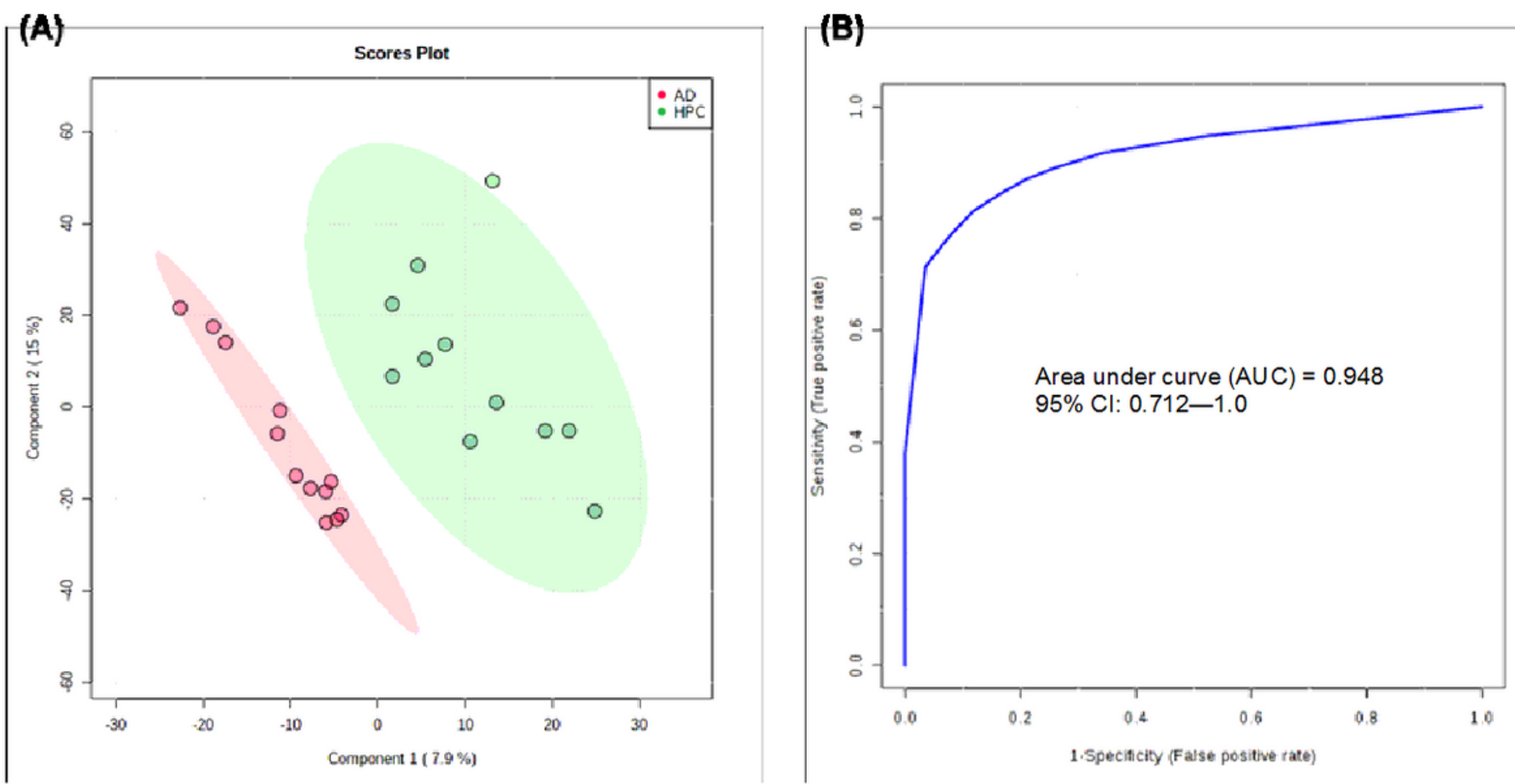

Figure 5 
(A) PLS-DA model constructed using levels of myristic acid, lauric acid, palmitic acid, and stearic acid for classification of AD and HPC groups, (B) ROC analysis of PLS-DA model by 100 -fold LOOCV showing $\mathrm{AUC}=0.948$.

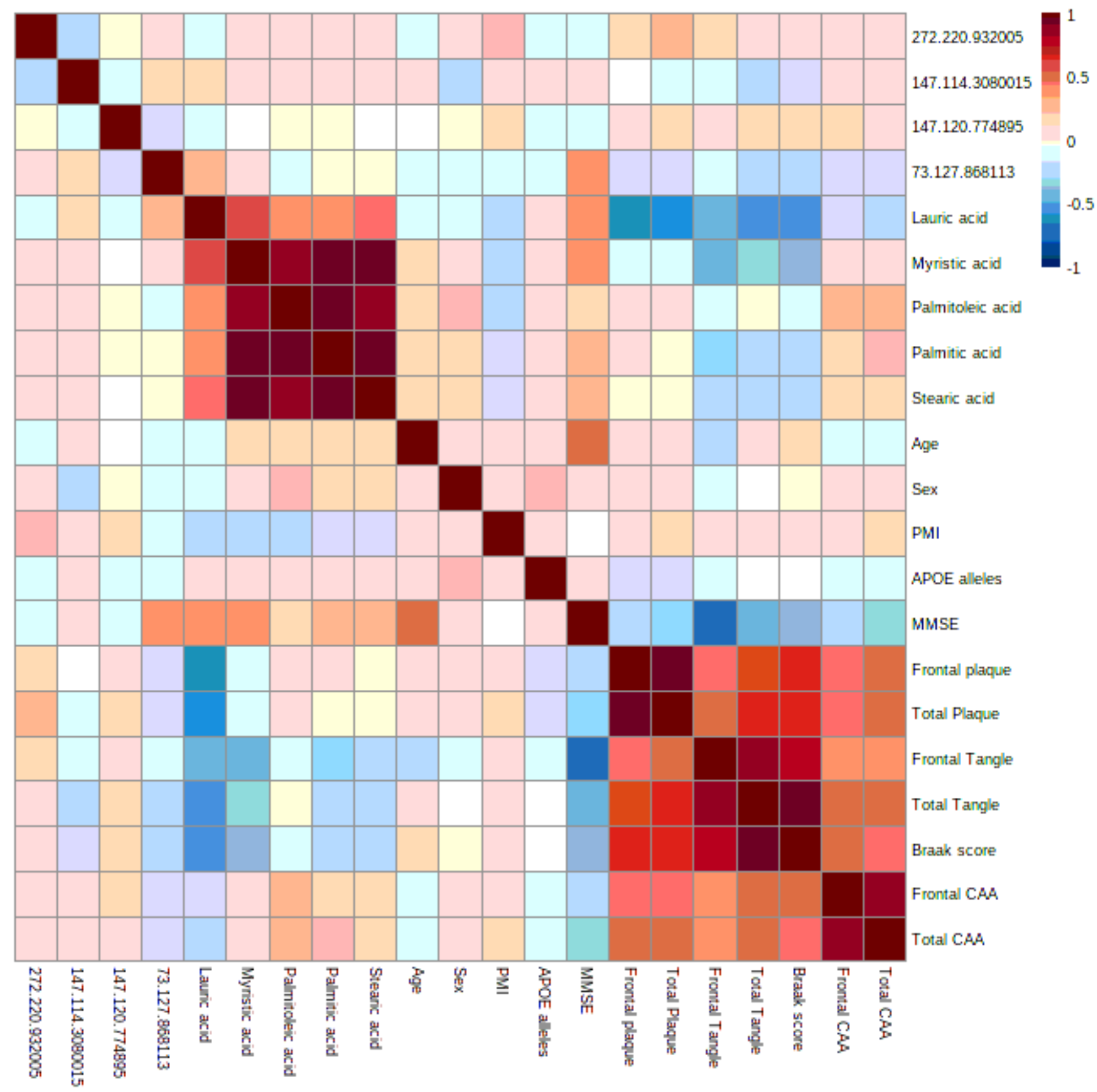

Figure 6

Correlation coefficients among the panel of candidate markers and clinical characteristics 


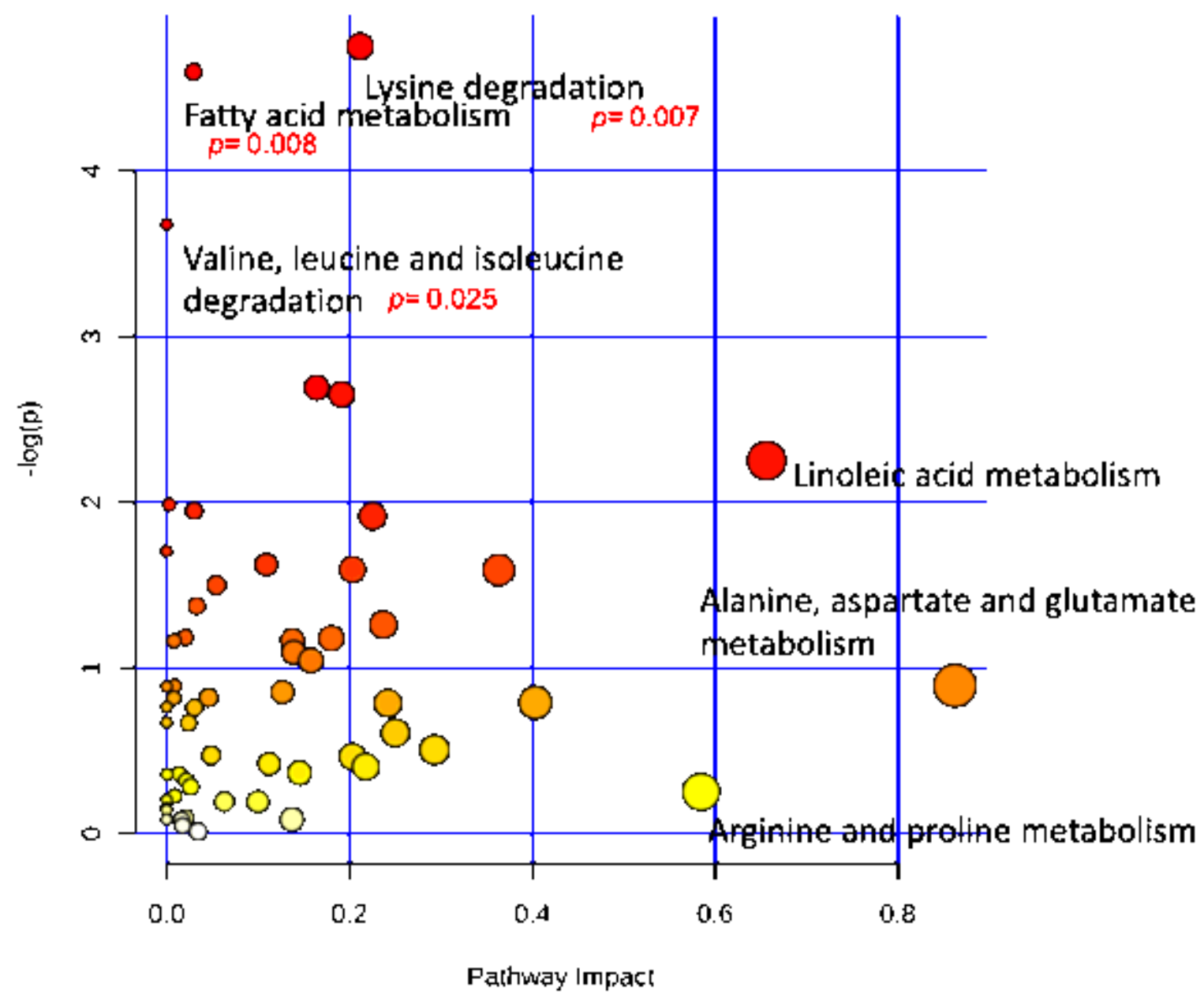

Figure 7

Metabolome view of pathway analysis conducted using levels of all reliably detected metabolites showing significantly altered pathways $(p<0.05)$ and those with high impact $(>0.50)$. 
$P$ value

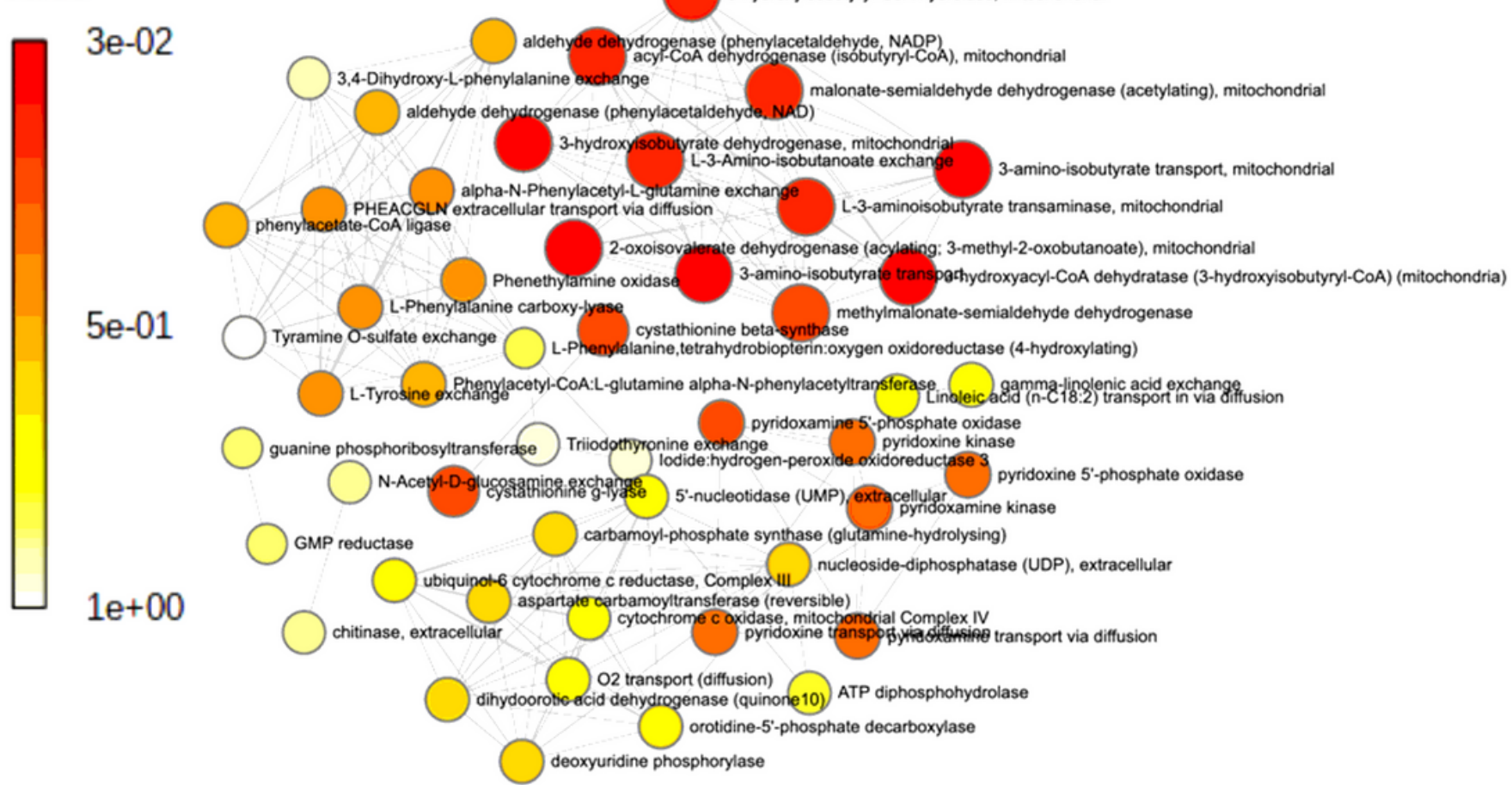

\section{Figure 8}

Network view of enzyme enrichment analysis conducted between case (MCl and AD) and control (NC and HPC). Enrichment ratios were significantly different for eleven enzymes between groups $(p<0.05)$.
NC
$\mathrm{HPC} / \mathrm{MCl}$
$\mathrm{MCI} / \mathrm{AD}$

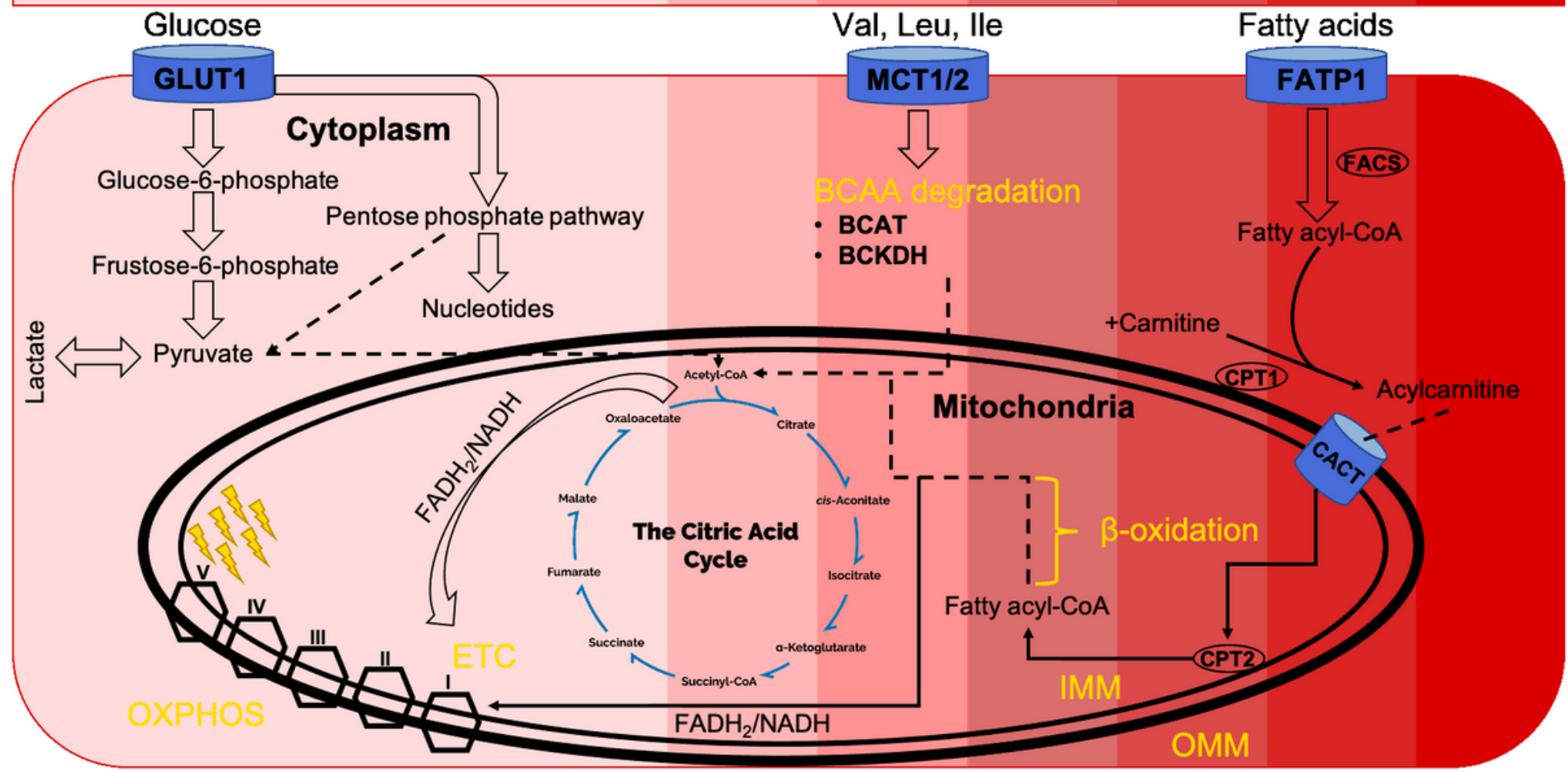




\section{Figure 9}

Conceptual schema articulating observed changes in substrate utilization and energy production as a function of increasing AD pathogenesis. Results show reduced aerobic glycolysis and increased degradation of BCAA associated with HPC and $\mathrm{MCl}$ groups, while a preference for fatty acid substrates is seen in $\mathrm{MCl}$ and $\mathrm{AD}$ groups.

\section{Supplementary Files}

This is a list of supplementary files associated with this preprint. Click to download.

- ADmetabolomicsSI.docx

- ADmetabolomicsSeparateExcelFileS1.xlsx 